TRANSACTIONS OF THE

AMERICAN MATHEMATICAL SOCIETY

Volume 354, Number 7 , Pages 2819-2848

S 0002-9947(02)02928-8

Article electronically published on February 14, 2002

\title{
REGULARITY PROPERTIES OF SOLUTIONS OF A CLASS OF ELLIPTIC-PARABOLIC NONLINEAR LEVI TYPE EQUATIONS
}

\author{
G. CITTI AND A. MONTANARI
}

\begin{abstract}
In this paper we prove the smoothness of solutions of a class of elliptic-parabolic nonlinear Levi type equations, represented as a sum of squares plus a vector field. By means of a freezing method the study of the operator is reduced to the analysis of a family $L_{\xi_{0}}$ of left invariant operators on a free nilpotent Lie group. The fundamental solution $\Gamma_{\xi_{0}}$ of the operator $L_{\xi_{0}}$ is used as a parametrix of the fundamental solution of the Levi operator, and provides an explicit representation formula for the solution of the given equation. Differentiating this formula and applying a bootstrap method, we prove that the solution is $C^{\infty}$.
\end{abstract}

\section{INTRODUCTION}

In this paper we study the regularity properties of solutions of a class of elliptic and parabolic equations, which contains in particular the motion by Levi curvature, and the prescribed Levi curvature equation. We denote by $\Omega$ an open set in $R^{3}$, by $\left(x_{1}, x_{2}, t\right)$ the elements of $R^{3}$, and for all $u: \Omega \rightarrow R$ we introduce the vector fields

$$
X_{u}=\partial_{x_{1}}+a \partial_{t}, \quad Y_{u}=\partial_{x_{2}}+b \partial_{t},
$$

where

$$
a=\frac{u_{x_{2}}-u_{x_{1}} u_{t}}{1+u_{t}^{2}} \text { and } b=-\frac{u_{x_{1}}+u_{x_{2}} u_{t}}{1+u_{t}^{2}} .
$$

Then the Levi operator is defined as

$$
\mathcal{L} u=X_{u}^{2} u+Y_{u}^{2} u-\left(X_{u} a+Y_{u} b\right) \partial_{t}
$$

and the Levi curvature of the graph of $u$ is

$$
\operatorname{curv}(u)=\frac{\mathcal{L} u}{\left(1+a^{2}+b^{2}\right)^{3 / 2}\left(1+u_{t}^{2}\right)^{1 / 2}} .
$$

Here we study the parabolic equation:

$$
\left.\mathcal{L} u+h(a, b, \cdot) \partial_{x_{3}} u=k\left(1+a^{2}+b^{2}\right)^{3 / 2}\left(1+u_{t}^{2}\right)^{1 / 2}, \quad \text { in } \Omega \times\right]-1,1[,
$$

where $k: \Omega \times]-1,1[\rightarrow \mathbb{R}$ and $h: \mathbb{R} \times \mathbb{R} \times \Omega \times]-1,1[$ are smooth. When the solution is independent of $x_{3}$, the equation becomes

$$
\mathcal{L} u=k\left(1+a^{2}+b^{2}\right)^{3 / 2}\left(1+u_{t}^{2}\right)^{1 / 2} \text { in } \Omega,
$$

Received by the editors May 3, 2000 and, in revised form, August 8, 2001.

2000 Mathematics Subject Classification. Primary 35J70, 35K65; Secondary 22E30.

Key words and phrases. Levi equation, elliptic-parabolic nonlinear equation, freezing method, Lie groups, fundamental solution, regularity properties.

Investigation supported by University of Bologna, founds for selected research topics.

(C)2002 American Mathematical Society 
which is, by (1.3), the equations of functions $u$ whose graph has at any point prescribed curvature $k$. If $k=0$ the equation becomes

$$
\left.\mathcal{L} u+h(a, b, \cdot) \partial_{x_{3}} u=0, \quad \text { in } \Omega \times\right]-1,1[,
$$

which describes the motion of the graph of $u$ by Levi curvature. We refer the reader to [ST1] and [ST2] for a survey of the geometric meaning and applications of these equations.

Existence results have been established for the solutions of (1.5) and (1.6), with different techniques. The Dirichlet problem for the stationary equation (1.5) has been studied by Bedford and Gaveau, who proved in [BG the existence of classical solutions, less regular than the boundary datum. For the same problem Slodkowsky and Tomassini proved in [ST1] the existence of a uniformly continuous solution under a requirement on the boundary datum weaker than that of [BG]. Analogous results hold for the existence of solutions of the Cauchy problem for the parabolic equation (1.6): Slodkowsky and Tomassini proved in [ST2] - [ST4 the existence of Lipschitz continuous solutions. This result was improved by Huisken and Klingenberg, who proved in $[\mathrm{HK}]$ the existence of a solution with the same regularity as the initial datum.

In all these papers the regularity of the solutions is not due to the equation, but is inherited from the regularity of the boundary data. On the other hand, here we study the interior regularity properties of the solutions, using in an essential way the structure of the operator and of the vector fields. A linear operator with the same structure as $\mathcal{L}+h \partial_{x_{3}}$ would be

$$
L_{L} u=X_{L}^{2} u+Y_{L}^{2} u-\left(X_{L} a+Y_{L} b+c\right) \partial_{t}+h \partial_{x_{3}},
$$

for linear vector fields $X_{L}=\partial_{x}+a \partial_{t}$ and $Y_{L}=\partial_{y}+b \partial_{t}$. When the vector fields are smooth, a sufficient condition for the hypoellipticity is the Hörmander condition (see $[\mathrm{H}]$ ), and in this case, the regularity of solutions has been studied by Folland $[\mathrm{F}$, Folland and Stein [FS], Rothschild and Stein [RS]. When the vector fields are not smooth, the Hörmander condition is meaningless, since the Lie algebra generated by $X_{L}$ and $Y_{L}$ is not defined. On the other hand, Franchi and Lanconelli in $\mathrm{FL}$ pointed out that the condition on the commutators can be replaced by the requirement that every pair of points $\xi$ and $\bar{\xi}$ can be connected by an integral curve of the vector fields. Under this assumption they proved a regularity result for solutions of a class of equations associated to linear nonsmooth operators defined in terms of suitable vector fields, which however does not contain the one studied here.

A different and more general technique for studying linear operators represented as in (1.7) was introduced in [C] and [CM1]. There, the authors proved a first regularity result for the stationary operator, in suitable classes $C_{L}^{m, \alpha}$ of Hölder continuous functions:

If $X_{L}, Y_{L},\left[X_{L}, Y_{L}\right]$ are linearly independent at every point and their coefficients $a, b, c$ belong to a suitable class of Hölder continuous functions $C_{L}^{m, \alpha}(\Omega)$, any solution $u \in C_{L}^{m-1, \alpha}(\Omega)$ of

$$
X_{L}^{2} u+Y_{L}^{2} u-\left(X_{L} a+Y_{L} b+c\right) \partial_{t} u=f \in C_{L}^{m-1, \alpha}(\Omega)
$$

is of class $C_{L}^{m+1, \alpha}(\Omega)$.

In $\mathrm{Csem}$ it was pointed out that the same theory applies to the more general equation (1.4), and that if the structure of the vector fields is rich, it could be 
convenient to study the solutions of the elliptic problem as stationary solutions of (1.4). Then, under the assumption that $X_{L}, Y_{L},\left[X_{L}, Y_{L}\right]$ are linearly independent at every point, the regularity result has been extended to the parabolic case by Montanari in [M]. We also refer to [CPP], where a simple presentation of the same method is provided, and it is applied to a different parabolic equation.

Due to the low regularity requirements on the coefficients of the operators, these kind of theorems can be naturally applied to nonlinear equations. It has been proved in $[\mathrm{C}$ that in the case of the Levi equation assumptions on the commutators can be expressed in terms of the curvature of the graph of $u$ :

$$
\left[X_{u}, Y_{u}\right]=-\frac{\mathcal{L} u}{1+u_{t}^{2}} \partial_{t}=-\frac{\operatorname{curv}(u)\left(1+a^{2}+b^{2}\right)^{3 / 2}}{\left(1+u_{t}^{2}\right)^{1 / 2}} \partial_{t}
$$

Hence, using the linear result, the following theorem has been proved in $[\mathrm{C}]$ :

Theorem. Assume that $k$ never vanishes in $\Omega$, and $u$ is a solution of (1.5) belonging to the standard euclidean class $C_{\text {euclid }}^{2, \alpha}(\Omega)$ with $\alpha>\frac{1}{2}$. Then it is of class $C^{\infty}(\Omega)$ (see [C] ).

The analogous linear parabolic result, applied to the Levi equation, holds (see [M]):

Theorem. If $k=0$, and $\operatorname{curv}(u)$ never vanishes in $\Omega \times]-1,1$, any solution $u$ of (1.6) of class $C_{L}^{2, \alpha}(\Omega \times]-1,1[)$ is of class $C^{\infty}(\Omega \times]-1,1[)$.

This method seems to work only when the curvature of $u$ never vanishes in $\Omega$. If the curvature is identically zero, different methods apply: foliation results for the graph of the solution have been established in [BG], [BK], [Sc], $\mathrm{CS}]$ with complex analysis techniques and in [CM1] by means of a real analysis method. However the solution is not regular as a function of all its derivatives, since any function of the variable $t$ is a solution.

On the other side, when the curvature has zeros of finite order the problem of the regularity was completely open, and its solution is the aim of this note. We will introduce a new technical apparatus, based on the property of the Lie algebra formally generated by $X_{u}$ and $Y_{u}$, which - as we will see - has step 3. As before we introduce a linear operator $L_{L}$ whose structure is formally the same as the Levi one, and study the regularity properties of its solutions. Applying these results to the nonlinear equation, we prove

Theorem 1.1. Assume that $u$ is a solution of class $C_{\text {euclid }}^{3, \alpha}$ of equation (1.4) in an open subset $\Omega \times]-1,1\left[\right.$ of $\mathbb{R}^{4}$, that $h \in C^{\infty}(\mathbb{R} \times \mathbb{R} \times \Omega \times]-1,1[)$, and that $k \in C^{\infty}(\Omega \times]-1,1[\times \mathbb{R})$. Assume that the following condition holds in $\left.\Omega \times\right]-1,1[$ :

$$
\operatorname{curv}^{2}(u)+\left(X_{u} \operatorname{curv}(u)\right)^{2}+\left(Y_{u} \operatorname{curv}(u)\right)^{2} \neq 0 .
$$

Then $u \in C^{\infty}(\Omega \times]-1,1[)$.

Obviously, by the results in $[\mathrm{C}$ and $[\mathrm{M}]$, we have only to study the regularity of the solution in a neighborhood of the points $\xi_{0}$, where $\operatorname{curv}(u)\left(\xi_{0}\right)=0$. At these points we can assume that $X_{u}(\operatorname{curv}(u))\left(\xi_{0}\right) \neq 0$. Due to condition (1.8) we also have

$$
\left[X_{u}, Y_{u}\right]_{\mid \xi_{0}}=0
$$


and

$$
\left[X_{u},\left[X_{u}, Y_{u}\right]\right]_{\mid \xi_{0}}=X_{u}(\operatorname{curv}(u))\left(\xi_{0}\right) \frac{\left(1+a^{2}+b^{2}\right)^{3 / 2}}{\left(1+u_{t}^{2}\right)^{1 / 2}} \partial_{t},
$$

so that $X_{u}, Y_{u}$, and $\left[X_{u},\left[X_{u}, Y_{u}\right]\right]$ are linearly independent at $\xi_{0}$, and, by continuity, in a neighborhood of this point. The choice of the last element of the basis, in terms of which we will define the control distance and the structure of the classes $C_{L}^{m, \alpha}$, is not unique. We will see that the apparently natural choice of $\partial_{x_{3}}$ does not properly describe the operator in our situation. The natural geometry associated to the operator is induced by the control distance generated by $X_{u}, Y_{u}$ and the vector field

$$
S_{u}=h \partial_{x_{3}}-\left(X_{u} a+Y_{u} b+c\right) \partial_{t} .
$$

Let us now state the linear result we need to prove Theorem 1.1 We fix an open set $\Omega \subset \mathbb{R}^{3}$, and functions

$$
\begin{gathered}
a, b \in C^{2}(\Omega \times]-1,1[), \quad c \in C^{2}(\Omega \times]-1,1[), \\
\left.h \in C^{2}(\Omega \times]-1,1[) \text { such that } 1 \leq h(\xi) \leq 2 \text { in } \Omega \times\right]-1,1[.
\end{gathered}
$$

We introduce, in analogy with (1.1) and (1.4), the linear vector fields

$$
X_{L}=\partial_{x_{1}}+a \partial_{t}, \quad Y_{L}=\partial_{x_{2}}+b \partial_{t}, \quad S_{L}=h \partial_{x_{3}}-\left(X_{L} a+Y_{L} b-c\right) \partial_{t},
$$

and the linear operator

$$
L_{L}=X_{L}^{2}+Y_{L}^{2}+S_{L}
$$

Then we study the regularity properties of solutions of

$$
\left.L_{L} u=g \text { in } \Omega \times\right]-1,1[.
$$

We assume that there exists $\left.\xi_{0} \in \Omega \times\right]-1,1[$ such that

$$
\left[X_{L}, Y_{L}\right]_{\mid \xi_{0}}=0, \quad\left[X_{L},\left[X_{L}, Y_{L}\right]\right]_{\mid \xi_{0}} \neq 0
$$

Since we are interested in a local result, we can assume that $\left[X_{L},\left[X_{L}, Y_{L}\right]\right]$ never vanishes in $\Omega \times]-1,1[$. Finally we assume that

$$
X_{L} a+Y_{L} b, \quad X_{L} b-Y_{L} a \in C^{2}(\Omega \times]-1,1[) .
$$

This last assumption, which seems to be very strong, is on the contrary very natural for our application, since, as we will see, it is satisfied by the nonlinear model.

In the following sections we will introduce suitable classes $C_{L}^{m, \alpha}(\Omega \times]-1,1[)$ of Hölder continuous functions and prove that

Theorem 1.2. Assume that the coefficients $a, b, h, c$ in equation (1.12) are of class

$$
C_{L}^{m-1, \alpha}(\Omega \times]-1,1[) \text {. }
$$

Assume that the second member $g$ is in $C_{L}^{m-2, \alpha}(\Omega \times]-1,1[)$, and that $\partial_{t} a, \partial_{t} b \in$ $C_{L}^{\alpha}(\Omega \times]-1,1[)$. Let $u \in C_{L}^{m-1, \alpha}(\Omega \times]-1,1[)$, be a solution of (1.12) such that $\partial_{t} u \in C_{L}^{1, \alpha}(\Omega \times]-1,1[), S_{L} u \in C_{L}^{1, \alpha}(\Omega \times]-1,1[)$. Then, $u \in C_{L}^{m, \beta}(\Omega \times]-1,1[)$, for every $\beta<\alpha$. 
The Lie algebra associated to the operator $L_{L}$ is not free up to step 3 at every point. Hence, we first develop a modified version of the well known method of Rothschild and Stein tailored to the equation at hand. We add some more variables and lift our vector fields to new vector fields $X, Y S$ such that the Lie algebra generated by $X, Y, S$ is free up to step 3 . The associated operator will be

$$
L=X^{2}+Y^{2}+S \text { in } \mathbb{R}^{8} .
$$

In Section 2 we describe the properties of this new operator in $R^{8}$, and also the properties of the associated classes $C_{L}^{m, \alpha}$ of Hölder continuous functions. Precisely, we will say that $X$ and $Y$ are "intrinsic" derivatives of weight $1, S$ of weight 2, and $\partial_{t}$, which is proportional to a third order commutator, of weight 3 . Derivatives of higher weight are defined accordingly. The classes $C_{L}^{m, \alpha}$ are the the spaces of functions whose derivatives of weight $m$ are Hölder continuous with respect to the control distance associated to the vector fields.

In Section 3 we will use a freezing method and we will associate to every point of the space a model operator $L_{\bar{\xi}}$ of Hörmander type, whose associated Lie algebra is nilpotent, free and, up to step 3, has the same structure as the one formally associated to $L$. This method is simpler than the procedure of Rothschild and Stein, because we directly define the frozen operator, by means of the Taylor polynomial of order 2 , in the direction of the vector fields. In this procedure the choice of the vector fields is crucial: the vector $S$ is defined in such a way that all the operators $L_{\xi_{0}}$ coincide, up to a change of variable. Then, by using a rather technical argument based on the Campbell-Hausdorff formula, we prove that the control distances $d_{\xi_{0}}$ and $d$ associated to $L_{\bar{\xi}}$ and $L$ are equivalent, and we study the dependence of $d_{\xi_{0}}$ on the variable $\xi_{0}$.

In Section 4 the fundamental solution of $L_{\bar{\xi}}$ will be used as a parametrix of the fundamental solution of $L$. Hence any solution of equation (1.4) admits an integral representation in terms of it and of suitable kernels $N_{\bar{\xi}, i}$ :

$$
u(\xi)=\sum_{i=1}^{3} \int \Gamma_{\bar{\xi}}(\xi, \zeta) N_{\bar{\xi}, i}(\zeta) d \zeta
$$

(see Theorem 4.3]. In [CPP] the authors provide necessary conditions on the kernels $N_{\bar{\xi}, i}$, which ensure the differentiability of the functions $u$. Similar conditions are satisfied in our context, and we can prove the regularity result stated in Theorem 1.2 .

In Section 5 we conclude the proof of Theorem 1.1, applying these results to the nonlinear equation. We first introduce a linearized operator

$$
L_{u}=X_{u}^{2}+Y_{u}^{2}-\left(X_{u} a+Y_{u} b+c\right) \partial_{t}+h \partial_{x_{3}},
$$

whose coefficients depend on $u$. In the classical bootstrap argument one differentiates the equation with respect to the spatial derivatives, and then deduces from this the regularity in the time direction. This procedure does not apply here because, if $u \in C_{L_{u}}^{m, \alpha}$, the function $X_{u} u$ is a solution of

$$
L_{u}\left(X_{u} u\right)=f\left(X_{u} u, \partial_{t} X_{u} u\right)
$$

where $f$ is a function of class $C^{\infty}$ of its arguments. Since the derivative with respect to $t$ has weight 3 , the second member is of class $C_{L_{u}}^{m-4, \alpha}$. Then $X_{u} u$ is of class $C_{L_{u}}^{m-2, \alpha}$ and $u$ is of class $C_{L_{u}}^{m-1, \alpha}$, which is less than the starting regularity. Hence we introduce a new bootstrap argument, using both euclidean and intrinsic 
derivatives. First we apply Theorem 1.2 and deduce that $u \in C_{L_{u}}^{7, \alpha}$. On the other side, if all the Euclidean derivatives $D^{m} u$ of order $m$ are of class $C_{L_{u}}^{7, \alpha}$, the function $\partial_{t} D^{m} u$ is a solution of

$$
L_{u}\left(\partial_{t} D^{m} u\right)=f\left(D^{m} u, X_{u} D^{m} u, Y_{u} D^{m} u\right),
$$

so that, again by Theorem 1.2 we deduce that $\partial_{t} D^{m} u \in C_{L_{u}}^{7, \alpha}$. Once the regularity in the direction $\partial_{t}$ is established, we can show in the same way that $\partial_{x_{3}} D^{m} u$, is of class $C_{L_{u}}^{7, \alpha}$, and then that $\partial_{x_{i}} D^{m} u \in C_{L_{u}}^{7, \alpha}$ with $i=1,2$. This obviously proves that the solution is smooth. We explicitly note that in this procedure the variable $t$ has a crucial role, while $x_{3}$ is treated as a spatial variable, and this explains the apparently nonstandard choice of its name.

\section{The Lifting PRocedure}

The behavior of the solutions of equations (1.12) is based on the structure of the Lie algebra generated by the vector fields $X_{L}, Y_{L}$ and $S_{L}$. By condition (1.13) we need commutators of weight at most 3 to generate the whole space, but the algebra is not free up to step 3. To overcome this problem we apply the lifting method, introduced by Rothschild and Stein in [RS], by adding suitable new variables, and lifting our original vector fields to new ones defined in $\mathbb{R}^{8}$. In this way the Lie algebra generated by the new vector fields $X, Y$ and $S$ is free up to step 3, i.e. $X, Y, S$ and their commutators up to weight 3 satisfy the minimal number of relations at every point and span the tangent space to $\mathbb{R}^{8}$. In this section we introduce a metric naturally associated to the vector fields, we define classes of Hölder continuous functions with respect to this metric, and we study the Taylor polynomials of a function belonging to these spaces.

The choice of new vector fields is not unique. We add the variables $\left(x_{4}, \cdots x_{7}\right)$, we order the components of each point in such a way that $t$ is the last variable and we consider the following vectors:

$$
\left\{\begin{array}{c}
X=X_{L}-x_{2} \partial_{x_{4}}-\frac{x_{2}^{2}}{2} \partial_{x_{7}} \\
Y=Y_{L}-x_{3} \partial_{x_{5}} \\
S=S_{L}+x_{1} \partial_{x_{6}} .
\end{array}\right.
$$

Correspondingly we can introduce a lifted operator

$$
L=X^{2}+Y^{2}+S
$$

and consider the equation

$$
L u=g \quad \text { in } U \subset \Omega \times]-1,1\left[\times R^{4} .\right.
$$

Remark 2.1. Assume that $u$ is a solution of (1.12) of class $C^{3}(\Omega \times]-1,1[)$. We can set $\widetilde{\xi}_{0}=\left(\xi_{0}, 0\right) \in U$; then the function $u$ can be trivially extended to a function (always denoted by $u$ ) that is a solution of (2.3) in $\Omega \times]-1,1\left[\times R^{4}\right.$ and independent of the new variables $x_{4}, \cdots, x_{7}$. Hence in the sequel we will study the more general equation (2.3).

In order to simplify notations we will denote

$$
D_{(0)}=\text { identity }, \quad D_{(1)}=X, \quad D_{(2)}=Y, \quad D_{(3)}=S .
$$

Also, for every multi-index $\sigma=\left(\sigma_{1}, \ldots, \sigma_{m}\right)$, with $\sigma_{r} \in\{0,1,2,3\}$, we set

$$
D_{\sigma}=D_{\sigma_{1}} \cdots D_{\sigma_{m}}
$$


and we define the weight of $\sigma$ and of $D_{\sigma}$ to be the natural number

$$
|(1)|=|(2)|=1, \quad|(3)|=2, \quad|\sigma|=\sum_{r=1}^{m}\left|\sigma_{r}\right| .
$$

The Lie derivative of a function $f$ with respect to a vector field $D_{\sigma}$ is defined as follows:

Definition 2.2. Let $U \subset \Omega \times]-1,1\left[\times R^{4}, f: U \rightarrow R, \bar{\xi} \in U\right.$, and let $\gamma$ be an integral curve of $D_{\sigma}$, starting at $\bar{\xi}$. If there exists

$$
\frac{d}{d h}(f \circ \gamma)_{\mid h=0},
$$

then we will denote it by $D_{\sigma} f(\bar{\xi})$, and we will call it derivative of $f$ in the direction $D_{\sigma}$ at the point $\bar{\xi}$.

A direct computation shows that the Lie algebra generated by $\left(D_{\sigma}\right)$ with $\sigma \in$ $\{(1),(2),(3)\}$ is free up to step 3 , and a possible choice of a basis is the following one:

$$
\begin{gathered}
D_{(4)}=[X, Y]=(X b-Y a) \partial_{t}+x_{2} \partial_{x_{7}}+\partial_{x_{4}}, \\
D_{(5)}=[Y, S]=Y h \partial_{x_{3}}-Y(X a+Y b-c) \partial_{t}-S b \partial_{t}+\partial_{x_{5}}, \\
D_{(6)}=[X, S]=X h \partial_{x_{3}}-X(X a+Y b-c) \partial_{t}-S a \partial_{t}+\partial_{x_{6}}, \\
D_{(7)}=[Y,[X, Y]]=\left(Y(X b-Y a)-(X b-Y a) \partial_{t} b\right) \partial_{t}+\partial_{x_{7}}, \\
D_{(8)}=[X,[X, Y]]=\left(X(X b-Y a)-(X b-Y a) \partial_{t} a\right) \partial_{t} .
\end{gathered}
$$

According to (2.5) we will also set

$$
|(4)|=2, \quad|(i)|=3, \quad \text { for } i=5, \cdots, 8 .
$$

Then we can define the canonical coordinates of any point, and a natural distance associated to these vector fields, as follows (see for example [NSW]):

Definition 2.3. If $\bar{\xi}$ belongs to $\Omega \times]-1,1\left[\times R^{4}\right.$, then the map

$$
F_{\bar{\xi}}: e \rightarrow \exp \left(\sum_{i=1}^{8} e_{i} D_{(i)}\right)(\bar{\xi})
$$

is a diffeomorphism of a neighborhood of the origin of $\mathbb{R}^{8}$ to a neighborhood $U_{\bar{\xi}}$ of $\bar{\xi}$ in $\mathbb{R}^{8}$. Its inverse function $\Phi_{D, \bar{\xi}}$ defines the canonical change of variable associated to the vector fields $D_{\sigma}$ and center $\bar{\xi}$. The numbers

$$
e=\Phi_{D, \bar{\xi}}(\xi)
$$

are called canonical coordinates of $\xi$ around $\bar{\xi}$ with respect to the vector fields $D_{(\sigma)}$.

By the local invertibility theorem, the open set $U_{\bar{\xi}}$ on which $\Phi_{D, \bar{\xi}}$ is defined depends continuously on $\bar{\xi}$. Hence in the sequel we will assume that $U$ is a fixed open set such that $U \subset \subset \Omega \times]-1,1\left[\times \mathbb{R}^{4}\right.$ and

$$
\exists \Phi_{D, \bar{\xi}}(\xi) \quad \forall \xi, \bar{\xi} \in U .
$$


According to the different weights defined in (2.5), it is natural to consider the homogeneous norm on $\mathbb{R}^{8}$

$$
\|e\|=\left(\left|e_{1}\right|^{N}+\left|e_{2}\right|^{N}+\left|e_{3}\right|^{N / 2}+\left|e_{4}\right|^{N / 2}+\sum_{i=5}^{8}\left|e_{i}\right|^{N / 3}\right)^{1 / N}
$$

where $N=18$. The natural distance induced by $\Phi_{D, \bar{\xi}}$ is defined as follows:

$$
\forall \xi, \bar{\xi} \in U d(\xi, \bar{\xi})=\left\|\Phi_{D, \bar{\xi}}(\xi)\right\| .
$$

In terms of this distance we can define natural Hölder continuous classes as follows:

Definition 2.4. Let $U$ be fixed as in (2.6). We say that $f \in C_{L}^{\alpha}(U)$ if there exists $C>0$ such that

$$
|f(\xi)-f(\bar{\xi})| \leq C d^{\alpha}(\xi, \bar{\xi}) \quad \forall \xi, \bar{\xi} \in U .
$$

We say that $f \in C_{i}^{\alpha}(U)$ if there exists a positive constant $C$ such that

$$
\left|f\left(\exp \left(h D_{(i)}\right)(\bar{\xi})\right)-f(\bar{\xi})\right| \leq C|h|^{\alpha},
$$

for every suitably small $h$, for every $\bar{\xi} \in U$.

We say that $u \in C_{L}^{1, \alpha}(U)$ if $X u, Y u \in C_{L}^{\alpha}(U)$ and $u \in C_{i}^{\frac{1+\alpha}{2}}(U)$, for $i=3$.

We say that $u \in C_{L}^{2, \alpha}(U)$ if $X u, Y u \in C_{L}^{1, \alpha}(U), S u \in C_{L}^{\alpha}(U)$.

Let $m \geq 3$ and let $a, b, h \in C_{L}^{m-1, \alpha}(U), c \in C_{L}^{m-2, \alpha}(U)$. We say that $u \in$ $C_{L}^{m, \alpha}(U)$ if $X u, Y u \in C_{L}^{m-1, \alpha}(U)$ and $S u \in C_{L}^{m-2, \alpha}(U)$.

For simplicity, in the sequel, when we write $u \in C_{L}^{m, \alpha}(U)$, for $m \geq 3$, we will assume implicitly that $a, b, h \in C_{L}^{m-1, \alpha}(U), c \in C_{L}^{m-2, \alpha}(U)$.

Let us recall the following properties of the classes $C_{L}^{m, \alpha}(U)$ :

Remark 2.5. If $u \in C_{L}^{1, \alpha}(U)$, then $u \in C_{i}^{\frac{1+\alpha}{3}}(U)$ for every $i=5, \cdots, 8$.

If $u \in C_{L}^{2, \alpha}(U)$, then $u \in C_{i}^{\frac{2+\alpha}{3}}(U)$, for every $i=5, \cdots, 8$.

Remark 2.6. If $0<\alpha<1$ and $m \geq 1$, then

$$
\begin{gathered}
\left.C_{L}^{m, \alpha}(U) \subseteq C_{L}^{m-1, \beta}(U), \quad \forall \beta \in\right] 0,1[. \\
C_{L}^{3 m, \alpha}(U) \subseteq C^{m, \frac{\alpha}{3}}(U) \subseteq C_{L}^{m, \frac{\alpha}{3}}(U),
\end{gathered}
$$

where $C^{m, \alpha}(U)$ is the space of functions with derivatives up to order $m$ that are $\alpha$-Hölder continuous functions in the Euclidean sense.

A function $f$ belonging to a class $C_{L}^{m, \alpha}(U)$ has a natural Taylor development.

Proposition 2.7. Let $U$ be fixed as in (2.6). Let $f \in C_{L}^{m, \alpha}(U)$, with $m=1,2$. Then $f$ has the following Taylor development of order 1 or 2 in the direction of the vector fields:

$$
f(\xi)=\widetilde{P}_{\bar{\xi}}^{m} f(\xi)+O\left(d^{m+\alpha}(\xi, \bar{\xi})\right), \quad m=1,2,
$$

where

$$
\widetilde{P}_{\bar{\xi}}^{1} f(\xi)=f(\bar{\xi})+\sum_{i=1}^{2} e_{i} D_{(i)} f(\bar{\xi})
$$




$$
\widetilde{P}_{\bar{\xi}}^{2} f(\xi)=f(\bar{\xi})+\sum_{i=1,}^{4} e_{i} D_{(i)} f(\bar{\xi})+\frac{1}{2} \sum_{i, j=1}^{2} e_{i} e_{j} D_{(i)} D_{(j)} f(\bar{\xi})
$$

and $\left(e_{1}, \ldots, e_{8}\right)$ are the canonical coordinates of $\xi$ around $\bar{\xi}$.

Proof. Let us first prove the assertion for $m=1$. Let $\xi, \bar{\xi}$ be fixed, and let

$$
e=\Phi_{D, \bar{\xi}}(\xi) \quad \text { and } \quad \xi_{2}=\exp \left(\sum_{i=1}^{2} e_{i} D_{(i)}\right)(\bar{\xi}) .
$$

Then

$$
\begin{gathered}
\xi=\exp \left(\sum_{i=1}^{8} e_{i} D_{(i)}\right)(\bar{\xi})=\exp \left(\sum_{i=1}^{8} e_{i} D_{(i)}\right) \exp \left(-\sum_{i=1}^{2} e_{i} D_{(i)}\right)\left(\xi_{2}\right) \\
=\exp \left(\sum_{i=3}^{8}\left(e_{i}+O\left(\|e\|^{2}\right)\right) D_{(i)}\right)\left(\xi_{2}\right) .
\end{gathered}
$$

Then, if $f \in C_{L}^{1, \alpha}(U)$,

$$
f(\xi)=f(\xi)-f\left(\xi_{2}\right)+f\left(\xi_{2}\right)-f(\bar{\xi})+f(\bar{\xi})
$$

(by the properties of the classes $C_{L}^{1, \alpha}(U)$ recalled in Remark 2.5, condition (2.8) and the definition of $\xi_{2}$ )

$$
=O\left(\sum_{i=3}^{8}\left(\left|e_{i}\right|^{(1+\alpha) / 2}+\|\left. e\right|^{(1+\alpha)}\right)\right)+f\left(\exp \left(\sum_{i=1}^{2} e_{i} D_{(i)}\right)(\bar{\xi})\right)-f(\bar{\xi})+f(\bar{\xi})
$$

(by definition of the distance $d$ and the mean value theorem)

$$
=f(\bar{\xi})+\sum_{i=1}^{2} e_{i} D_{(i)} f(\widetilde{\xi})+O\left(d^{1+\alpha}(\xi, \bar{\xi})\right)
$$

(where $\widetilde{\xi}$ satisfies $d(\widetilde{\xi}, \bar{\xi}) \leq d\left(\xi_{2}, \bar{\xi}\right) \leq d\left(\xi_{2}, \xi\right)+d(\xi, \bar{\xi}) \leq 2 d(\xi, \bar{\xi})$, and since $D_{(i)} f$ is Hölder continuous)

$$
=P_{\bar{\xi}}^{1} f(\xi)+O\left(d^{1+\alpha}(\xi, \bar{\xi})\right) .
$$

The proof for $m=2$ is similar to the preceding one. We choose

$$
\xi_{3}=\exp \left(\sum_{i=3}^{4} e_{i} D_{(i)}\right)\left(\xi_{2}\right)
$$

and as before we get

$$
\begin{gathered}
\xi=\exp \left(\sum_{i=1}^{8} e_{i} D_{(i)}\right)(\bar{\xi}) \\
=\exp \left(\sum_{i=1}^{8} e_{i} D_{(i)}\right) \exp \left(-\sum_{i=1}^{2} e_{i} D_{(i)}\right) \exp \left(-\sum_{i=3}^{4} e_{i} D_{(i)}\right)\left(\xi_{3}\right) \\
=\exp \left(\sum_{i=5}^{8}\left(e_{i}+O\left(\|e\|^{3}\right)\right) D_{(i)}\right)\left(\xi_{3}\right)
\end{gathered}
$$


We immediately deduce that

$$
f(\xi)=f(\xi)-f\left(\xi_{3}\right)+f\left(\xi_{3}\right)-f\left(\xi_{2}\right)+f\left(\xi_{2}\right)-f(\bar{\xi})+f(\bar{\xi})
$$

(by Remark 2.5 and condition (2.9))

$$
\begin{gathered}
=O\left(\sum_{i=5}^{8}\left|e_{i}\right|^{(2+\alpha) / 3}+\|\left. e\right|^{2+\alpha}\right)+f\left(\exp \left(\sum_{i=3}^{4} e_{i} D_{(i)}\right)\left(\xi_{2}\right)\right)-f\left(\xi_{2}\right) \\
+f\left(\exp \left(\sum_{i=1}^{2} e_{i} D_{(i)}\right)(\bar{\xi})\right)-f(\bar{\xi})+f(\bar{\xi})
\end{gathered}
$$

(by the mean value theorem and (2.9))

$$
\begin{aligned}
=f(\bar{\xi})+ & \sum_{i=1}^{2} e_{i} D_{(i)} f(\bar{\xi})+\frac{1}{2} \sum_{i j,=1}^{2} e_{i} e_{j} D_{(i)} D_{(j)} f\left(\widetilde{\xi}_{2}\right) \\
& +\sum_{i=3}^{4} e_{i} D_{(i)} f\left(\widetilde{\xi}_{3}\right)+O\left(d^{2+\alpha}(\xi, \bar{\xi})\right)
\end{aligned}
$$

(since the derivatives of $f$ are Hölder continuous)

$$
=\widetilde{P}_{\bar{\xi}}^{2} f(\xi)+O\left(d^{2+\alpha}(\xi, \bar{\xi})\right) .
$$

Note that $\widetilde{P}_{\bar{\xi}}^{2} f$ is a polynomial in the canonical coordinates. If we need a polynomial in the Euclidean coordinates, we have to approximate the canonical coordinates $e_{i}$ of a point $\xi$ in a neighborhood of $\bar{\xi}$ with polynomials.

Remark 2.8. If $f \in C_{L}^{2, \alpha}(U)$, we have

$$
f(\xi)=P_{\bar{\xi}}^{2} f(\xi)+O\left(d^{2+\alpha}(\xi, \bar{\xi})\right),
$$

where $P_{\bar{\xi}}^{2} f(\xi)$ is the polynomial in the Euclidean coordinates

$$
P_{\bar{\xi}}^{2} f(\xi)=f(\bar{\xi})+\sum_{i=1, i \neq 3}^{4} e_{i} D_{(i)} f(\bar{\xi})+\frac{x_{3}-\bar{x}_{3}}{h(\bar{\xi})} D_{(3)} f(\bar{\xi})+\frac{1}{2} \sum_{i, j=1}^{2} e_{i} e_{j} D_{(i)} D_{(j)} f(\bar{\xi}) .
$$

Besides,

$$
\left|P_{\bar{\xi}}^{2} f(\xi)-P_{\xi_{1}}^{2} f(\xi)\right| \leq C\left(d^{2+\alpha}\left(\bar{\xi}, \xi_{1}\right)+d^{\alpha}\left(\bar{\xi}, \xi_{1}\right) d^{2}(\bar{\xi}, \xi)\right) .
$$

If $f \in C_{L}^{3, \alpha}(U)$, or $f \in C_{\text {euclidean }}^{2, \alpha}(U)$ and $\xi=\exp (s S)(\bar{\xi})$, then

$$
\left|P_{\bar{\xi}}^{2} f(\xi)-P_{\xi_{1}}^{2} f(\xi)\right| \leq C s^{(2+\alpha) / 2}+C s^{(1+\alpha) / 2} d(\bar{\xi}, \xi) .
$$

Proof. We first note that $P_{\bar{\xi}}^{2} f$ is a polynomial in the Euclidean coordinates. Indeed,

$$
\begin{gathered}
e_{1}=x_{1}-\bar{x}_{1}, \quad e_{2}=x_{2}-\bar{x}_{2}, \\
e_{4}=x_{4}-\bar{x}_{4}+\left(x_{1}-\bar{x}_{1}\right) \bar{x}_{2}+\frac{\left(x_{1}-\bar{x}_{1}\right)\left(x_{2}-\bar{x}_{2}\right)}{2} .
\end{gathered}
$$

Besides, the first assertion is a consequence of Proposition [2.7, since

$$
e_{3}=\frac{x_{3}-\bar{x}_{3}}{h(\bar{\xi})}+O\left(d(\xi, \bar{\xi})^{3}\right) .
$$


We also have, for every $i=1, \ldots, 4$ :

$$
D_{(i)} P_{\bar{\xi}}^{2} f=P_{\bar{\xi}}^{2-|i|} D_{(i)} f .
$$

Hence if we set

$$
F(\xi)=P_{\bar{\xi}}^{2} f(\xi)-P_{\xi_{1}}^{2} f(\xi)
$$

we get

$$
\begin{gathered}
P_{\bar{\xi}}^{2} f(\xi)-P_{\xi_{1}}^{2} f(\xi)=F(\xi) \\
=F(\bar{\xi})+\sum_{i=1}^{4} e_{i}(\xi, \bar{\xi}) D_{(i)} F(\bar{\xi})+\frac{1}{2} \sum_{i, j=1}^{2} e_{i}(\xi, \bar{\xi}) e_{j}(\xi, \bar{\xi}) D_{(i)} D_{(j)} F(\bar{\xi}) \\
=f(\bar{\xi})-P_{\xi_{1}}^{2} f(\bar{\xi})+\sum_{i=1}^{2} e_{i}(\xi, \bar{\xi})\left(D_{(i)} f(\bar{\xi})-P_{\xi_{1}}^{1} D_{(i)} f(\bar{\xi})\right) \\
+\sum_{i=3}^{4} e_{i}(\xi, \bar{\xi})\left(D_{(i)} f(\bar{\xi})-D_{(i)} f\left(\xi_{1}\right)\right) \\
+\frac{1}{2} \sum_{i, j=1}^{2} e_{i}(\xi, \bar{\xi}) e_{j}(\xi, \bar{\xi})\left(D_{(i)} D_{(j)} f(\bar{\xi})-D_{(i)} D_{(j)} f\left(\xi_{1}\right)\right) \\
\leq C\left(d^{2+\alpha}\left(\bar{\xi}, \xi_{1}\right)+d^{\alpha}\left(\bar{\xi}, \xi_{1}\right) d^{2}(\bar{\xi}, \xi)\right)
\end{gathered}
$$

and this proves (2.10). If $f \in C_{L}^{3, \alpha}(U)$ or $f \in C_{\text {euclidean }}^{2, \alpha}(U)$ and $\xi_{1}=\exp (s S)(\bar{\xi})$, then

$$
\left|D_{i} f(\bar{\xi})-D_{i} f\left(\xi_{1}\right)\right|+\left|D_{i, j} f(\bar{\xi})-D_{i, j} f\left(\xi_{1}\right)\right| \leq C s^{(1+\alpha) / 2} .
$$

Inserting this estimate in the preceding inequality, we deduce assertion (2.11).

\section{The FreEZING PROCEDURE}

In this section we introduce new vector fields $X_{\bar{\xi}}, Y_{\bar{\xi}}$, and $S_{\bar{\xi}}$, whose generated Lie algebra is nilpotent and has the same structure as the Lie algebra generated by $X, Y$, and $S$. Since we need to compute commutators of weight 3 of $X$ and $Y$, we define our frozen vector fields, substituting for the coefficients $a$ and $b$ their Taylor expansion of second order, while we only have to compute second order commutators of $S$, so we will consider first order development of its coefficient. With this choice of polynomial we define the vector fields as follows:

$$
\left\{\begin{array}{c}
X_{\bar{\xi}}=\partial_{x_{1}}+P_{\bar{\xi}}^{2} a \partial_{t}-x_{2} \partial_{x_{4}}-\frac{x_{2}^{2}}{2} \partial_{x_{7}}, \\
Y_{\bar{\xi}}=\partial_{x_{2}}+P_{\bar{\xi}}^{2} b \partial_{t}-x_{3} \partial_{x_{5}}, \\
S_{\bar{\xi}}=P_{\bar{\xi}}^{1} h \partial_{x_{3}}-P_{\bar{\xi}}^{1}(X a+Y b-c) \partial_{t}+x_{1} \partial_{x_{6}} .
\end{array}\right.
$$

The frozen operator of the operator $L$ defined in (2.2) will be defined in terms of the new vector fields $X_{\bar{\xi}}, Y_{\bar{\xi}}$ and $S_{\bar{\xi}}$ as follows:

$$
L_{\bar{\xi}}=X_{\bar{\xi}}^{2}+Y_{\bar{\xi}}^{2}+S_{\bar{\xi}} \text {. }
$$

$L_{\bar{\xi}}$ is a $C^{\infty}$ operator satisfying the Hörmander condition; hence there exist a control distance $d_{\bar{\xi}}$ and a fundamental solution $\Gamma_{\bar{\xi}}$ associated to $L_{\bar{\xi}}$. Moreover, the 
homogeneous dimension of the space is $N=18$, and $\Gamma_{\bar{\xi}}$ is locally equivalent to $d_{\bar{\xi}}^{-N+2}$. Let us now study in detail the properties of the distance $d_{\bar{\xi}}$, and of the fundamental solution.

In order to simplify notations we introduce notations similar to (2.4):

$$
D_{\bar{\xi},(0)}=\text { identity }, \quad D_{\bar{\xi},(1)}=X_{\bar{\xi}}, \quad D_{\bar{\xi},(2)}=Y_{\bar{\xi}}, \quad D_{\bar{\xi},(3)}=S_{\bar{\xi}}
$$

Remark 3.1. For the reader's convenience we compute the commutators of the frozen vector fields. From their explicit expression the structure of the generated Lie algebra will be clear. We have

$$
\begin{gathered}
D_{\bar{\xi},(4)}=\left[X_{\bar{\xi}}, Y_{\bar{\xi}}\right]=\left(P_{\bar{\xi}}^{1}(X b)-P_{\bar{\xi}}^{1}(Y a)\right) \partial_{t}+x_{2} \partial_{x_{7}}+\partial_{x_{4}}, \\
D_{\bar{\xi},(5)}=\left[Y_{\bar{\xi}}, S_{\bar{\xi}}\right]=Y h(\bar{\xi}) \partial_{x_{3}}-Y(X a+Y b-c)(\bar{\xi}) \partial_{t}-S b(\bar{\xi}) \partial_{t}+\partial_{x_{5}}, \\
D_{\bar{\xi},(6)}=\left[X_{\bar{\xi}}, S_{\bar{\xi}}\right]=X h(\bar{\xi}) \partial_{x_{3}}-X(X a+Y b-c)(\bar{\xi}) \partial_{t}-S a(\bar{\xi}) \partial_{t}+\partial_{x_{6}}, \\
D_{\bar{\xi},(7)}=\left[Y_{\bar{\xi}},\left[X_{\bar{\xi}}, Y_{\bar{\xi}}\right]\right]=\left(Y(X b-Y a)(\bar{\xi})-(X b-Y a) \partial_{t} b(\bar{\xi})\right) \partial_{t}+\partial_{x_{7}}, \\
D_{\bar{\xi},(8)}=\left[X_{\bar{\xi}},\left[X_{\bar{\xi}}, Y_{\bar{\xi}}\right]\right]=\left(X(X b-Y a)(\bar{\xi})-(X b-Y a) \partial_{t} a(\bar{\xi})\right) \partial_{t} .
\end{gathered}
$$

From this it follows that the Lie algebra generated by $X_{\bar{\xi}}, Y_{\bar{\xi}}$ and $S_{\bar{\xi}}$ is a free nilpotent Lie algebra with three generators and of step three. The vectors $\left(D_{\bar{\xi},(i)}\right)$ are a basis of the vector space underlying the Lie algebra. Hence the exponential mapping

$$
F_{\bar{\xi}, \xi}: e \longrightarrow \exp \left(\sum_{i=1}^{8} e_{i} D_{\bar{\xi},(i)}\right)(\xi)
$$

is a global diffeomorphism. Its inverse function, the canonical change of variable associated to the vector fields $D_{\bar{\xi}}$ and center $\xi$, will be denoted

$$
\Phi_{\bar{\xi}, \xi}, \quad \text { and if } \xi=\bar{\xi}, \quad \text { then } \Phi_{\bar{\xi}}=\Phi_{\bar{\xi}, \bar{\xi}} \text {. }
$$

The control distance $d_{\bar{\xi}}$ associated to $D_{\bar{\xi},(i)}$ is equivalent to the quasi distance (see [NSW]

$$
d_{\bar{\xi}}(\xi, \bar{\xi})=\left\|\Phi_{\bar{\xi}}(\xi)\right\| .
$$

Proposition 3.2. There exist two constants $C_{1}$ and $C_{2}$ such that

$$
C_{1} d(\bar{\xi}, \xi) \leq d_{\bar{\xi}}(\bar{\xi}, \xi) \leq C_{2} d(\bar{\xi}, \xi)
$$

for all $\xi, \bar{\xi} \in U$.

Proof. Let us write $e=\Phi_{D, \bar{\xi}}(\xi)$ and $\bar{e}=\Phi_{\bar{\xi}}(\xi)$. In order to estimate $e$ and $\bar{e}$, we consider the following two curves:

$$
\gamma(s)=\exp \left(s \sum_{i=1}^{8} e_{i} D_{(i)}\right)(\bar{\xi}) \quad \text { and } \quad \bar{\gamma}(s)=\exp \left(s \sum_{i=1}^{8} \bar{e}_{i} D_{\bar{\xi},(i)}\right)(\bar{\xi}) .
$$

Then the first components satisfy the following equations:

$$
\gamma_{1}^{\prime}(s)-\bar{\gamma}_{1}^{\prime}(s)=e_{1}-\bar{e}_{1}, \quad\left(\gamma_{1}-\bar{\gamma}_{1}\right)(0)=\left(\gamma_{1}-\bar{\gamma}_{1}\right)(1)=0
$$


Hence

$$
e_{1}=\bar{e}_{1} .
$$

Analogously,

$$
e_{2}=\bar{e}_{2} \quad \text { and } \quad e_{4}=\bar{e}_{4} .
$$

Let us consider the third component:

$$
\begin{gathered}
\gamma_{3}^{\prime}(s)=e_{3} h(\gamma)+e_{5} Y h(\gamma)+e_{6} X h(\gamma), \\
\bar{\gamma}_{3}^{\prime}(s)=\bar{e}_{3} P_{\bar{\xi}}^{1} h(\bar{\gamma})+\bar{e}_{5} Y h(\bar{\xi})+\bar{e}_{6} X h(\bar{\xi}) .
\end{gathered}
$$

Integrating, we get

$$
\begin{gathered}
\left|e_{3}-\bar{e}_{3}\right|\left|\int_{0}^{1} h(\gamma(s)) d s\right| \\
\leq\left|\bar{e}_{3}\right| \int_{0}^{1}\left|h(\gamma(s))-P_{\bar{\xi}}^{1} h(\bar{\gamma}(s))\right| d s+\left|\bar{e}_{5}\right| \int_{0}^{1}|Y h(\gamma(s))-Y h(\bar{\xi})| d s \\
+\left|\bar{e}_{5}-e_{5}\right|+\left|\bar{e}_{6}\right| \int_{0}^{1}|X h(\gamma(s))-X h(\bar{\xi})| d s+\left|\bar{e}_{6}-e_{6}\right| .
\end{gathered}
$$

Since $P_{\bar{\xi}}^{1} h$ depends on the first two coordinates of its argument, which coincide in $\gamma$ and $\bar{\gamma}$, it follows that $P_{\bar{\xi}}^{1} h(\gamma(s))=P_{\bar{\xi}}^{1} h(\bar{\gamma}(s))$. The function $h$ is of class $C_{\text {euclid }}^{2}$ in the Euclidean sense; hence it belongs to $C_{L}^{1, \alpha}$ and, by Proposition 2.7 .

$$
\left|h(\gamma(s))-P_{\bar{\xi}}^{1} h(\bar{\gamma}(s))\right|=\left|h(\gamma(s))-P_{\bar{\xi}}^{1} h(\gamma(s))\right| \leq C d^{1+\alpha}(\gamma(s), \bar{\xi}) \leq C d^{1+\alpha}(\xi, \bar{\xi}) .
$$

Analogously, since $X h$ and $Y h$ are of class $C_{\text {euclid }}^{1}$, then

$$
|X h(\gamma(s))-X h(\bar{\xi})|+|Y h(\gamma(s))-Y h(\bar{\xi})| \leq C d(\gamma(s), \bar{\xi}) \leq C d(\xi, \bar{\xi}),
$$

for a suitable constant $C$. Inserting in (3.6) the previous estimates and using the fact that $h \geq 1$, we get

$$
\begin{gathered}
\left|e_{3}-\bar{e}_{3}\right| \leq\left|e_{3}-\bar{e}_{3}\right|\left|\int_{0}^{1} h(\gamma(s)) d s\right| \\
\leq\left|\bar{e}_{3}\right| d^{1+\alpha}(\xi, \bar{\xi})+\left(\left|\bar{e}_{5}\right|+\left|\bar{e}_{6}\right|\right) d(\xi, \bar{\xi})+\left|\bar{e}_{5}-e_{5}\right|+\left|\bar{e}_{6}-e_{6}\right| \\
\leq d_{\bar{\xi}}^{2}(\bar{\xi}, \xi) d^{1+\alpha}(\bar{\xi}, \xi)+d_{\bar{\xi}}^{2+\alpha}(\bar{\xi}, \xi) d(\bar{\xi}, \xi)+\left|\bar{e}_{5}-e_{5}\right|+\left|\bar{e}_{6}-e_{6}\right| .
\end{gathered}
$$

Let us consider the fifth component:

$$
\gamma_{5}^{\prime}(s)=-e_{2} \gamma_{3}(s)+e_{5}, \quad \bar{\gamma}_{5}^{\prime}(s)=-\bar{e}_{2} \bar{\gamma}_{3}(s)+\bar{e}_{5} .
$$

Integrating and using the fact that $e_{2}=\bar{e}_{2}$, we get

$$
\begin{aligned}
\mid \bar{e}_{5}- & e_{5}|=| e_{2}\left|\int_{0}^{1}\right| \gamma_{3}(s)-\bar{\gamma}_{3}(s)|d s \leq| e_{2}\left|\int_{0}^{1} \int_{0}^{s}\right| \gamma_{3}^{\prime}(\tau)-\bar{\gamma}_{3}^{\prime}(\tau) \mid d \tau d s \\
\leq & \left|e_{2}\right|\left(\left|e_{3}-\bar{e}_{3}\right|+\left|\bar{e}_{3}\right| \int_{0}^{1}\left|h(\gamma(\tau))-P_{\bar{\xi}}^{1} h(\bar{\gamma}(\tau))\right| d \tau\right) \\
& +\left|e_{2}\right|\left(\left|e_{5}\right|+\left|e_{6}\right|\right)+\left|e_{2}\right|\left(\left|\bar{e}_{5}\right|+\left|\bar{e}_{6}\right|\right) \leq
\end{aligned}
$$


(by (3.7))

$$
\begin{aligned}
& \leq\left|e_{2}\right|\left|e_{3}-\bar{e}_{3}\right|+\left|e_{2}\right|\left|\bar{e}_{3}\right| d^{1+\alpha}(\xi, \bar{\xi})+\left|e_{2}\right|\left(\left|e_{5}\right|+\left|e_{6}\right|+\left|\bar{e}_{5}\right|+\left|\bar{e}_{6}\right|\right) \\
& \quad \leq\left|e_{2}\right|\left|e_{3}-\bar{e}_{3}\right|+d_{\bar{\xi}}(\xi, \bar{\xi}) d^{2+\alpha}(\xi, \bar{\xi})+d(\xi, \bar{\xi}) d_{\bar{\xi}}^{2+\alpha}(\xi, \bar{\xi}) .
\end{aligned}
$$

Analogously,

$$
\left|\bar{e}_{6}-e_{6}\right| \leq\left|e_{1}\right|\left|e_{3}-\bar{e}_{3}\right|+d_{\bar{\xi}}(\xi, \bar{\xi}) d^{2+\alpha}(\xi, \bar{\xi})+d(\xi, \bar{\xi}) d_{\bar{\xi}}^{2+\alpha}(\xi, \bar{\xi}) .
$$

Inserting this estimate in (3.8), we get, for $e_{1}, e_{2}$ sufficiently small,

$$
\left|\bar{e}_{3}-e_{3}\right| \leq d(\xi, \bar{\xi}) d_{\bar{\xi}}^{2+\alpha}(\xi, \bar{\xi})+d^{2+\alpha}(\xi, \bar{\xi}) d_{\bar{\xi}}(\xi, \bar{\xi}) .
$$

We can now consider the last component:

$$
\begin{gathered}
\left|e_{8}-\bar{e}_{8}\right| \leq e_{1} \int_{0}^{1}\left|a(\gamma(s))-P_{\bar{\xi}}^{2} a(\bar{\gamma}(s))\right| d s \\
+e_{2} \int_{0}^{1}\left|b(\gamma(s))-P_{\bar{\xi}}^{2} b(\bar{\gamma}(s))\right| d s \\
+\bar{e}_{3} \int_{0}^{1}\left|\widetilde{c}(\gamma(s))-P_{\bar{\xi}}^{1} \widetilde{c}(\bar{\gamma}(s))\right| d s+\left|e_{3}-\bar{e}_{3}\right| \\
+e_{4} \int_{0}^{1}\left|d(\gamma(s))-P_{\bar{\xi}}^{1} d(\bar{\gamma}(s))\right| d s \\
+O\left(\left|e_{5}\right|+\left|e_{6}\right|+\left|e_{7}\right|\right)+O\left(\left|\bar{e}_{5}\right|+\left|\bar{e}_{6}\right|+\left|\bar{e}_{7}\right|\right),
\end{gathered}
$$

where $\widetilde{c}=-(X a+Y b-c)$, and $d=X b-Y a$. Using again the fact that $P_{\bar{\xi}}^{1}$ depends only on the first two coordinates, and the properties of the Taylor polynomial,

$$
\begin{gathered}
\left|e_{8}-\bar{e}_{8}\right| \leq d_{\bar{\xi}}(\xi, \bar{\xi}) \int_{0}^{1} d^{2+\alpha}(\gamma(s), \bar{\xi}) d s+d_{\bar{\xi}}^{2}(\xi, \bar{\xi}) \int_{0}^{1} d^{1+\alpha}(\gamma(s), \bar{\xi}) d s \\
+O\left(\left|e_{5}\right|+\left|e_{6}\right|+\left|e_{7}\right|\right)+O\left(\left|\bar{e}_{5}\right|+\left|\bar{e}_{6}\right|+\left|\bar{e}_{7}\right|\right)+\left|e_{3}-\bar{e}_{3}\right| .
\end{gathered}
$$

Then

$$
\begin{gathered}
\left|\bar{e}_{8}-e_{8}\right| \leq d(\xi, \bar{\xi}) d_{\bar{\xi}}^{2}(\xi, \bar{\xi})+d^{2}(\xi, \bar{\xi}) d_{\bar{\xi}}(\xi, \bar{\xi}) \\
+O\left(\left|e_{5}\right|+\left|e_{6}\right|+\left|e_{7}\right|\right)+O\left(\left|\bar{e}_{5}\right|+\left|\bar{e}_{6}\right|+\left|\bar{e}_{7}\right|\right)+\left|e_{3}-\bar{e}_{3}\right| .
\end{gathered}
$$

Finally,

$$
\bar{e}_{7}=e_{7} .
$$

From the estimates (3.4), (3.5), (3.13), and the definition of the distance, we get

$$
d(\xi, \bar{\xi}) \leq d_{\bar{\xi}}(\xi, \bar{\xi})+\left|e_{3}-\bar{e}_{3}\right|^{1 / 2}+\left|e_{5}-\bar{e}_{5}\right|^{1 / 3}+\left|e_{6}-\bar{e}_{6}\right|^{1 / 3}+\left|e_{8}-\bar{e}_{8}\right|^{1 / 3}
$$

(by (3.11), (3.9), (3.10), 3.12)

$$
\leq d_{\bar{\xi}}(\xi, \bar{\xi})+d_{\bar{\xi}}^{1 / 3}(\xi, \bar{\xi}) d^{2 / 3}(\xi, \bar{\xi})+d^{1 / 3}(\xi, \bar{\xi}) d_{\bar{\xi}}^{2 / 3}(\xi, \bar{\xi}) .
$$


Now the first inequality of the thesis follows. The other inequality follows in the same way from the established estimates.

By construction, the Lie algebra generated by $X_{\bar{\xi}}, Y_{\bar{\xi}}$ and $S_{\bar{\xi}}$ has the same structure at every point. Hence the canonical change of variables changes $X_{\bar{\xi}}$, $Y_{\bar{\xi}}$ and $S_{\bar{\xi}}$ into linear vector fields of class $C^{\infty}$, nilpotent and independent of $\bar{\xi}$. Precisely, we have

Remark 3.3. We say that a vector field $\bar{D}_{(i)}$ is the image of $D_{\bar{\xi},(i)}$ with respect to the canonical change of variables defined in (3.3), if for every function $f \in C_{L}^{1}(U)$

$$
\bar{D}_{(i)} f_{\bar{\xi}}=D_{\bar{\xi},(i)} f, \quad \text { where } f_{\bar{\xi}}=f \circ \Phi_{\bar{\xi}}^{-1} .
$$

If $\Phi_{\bar{\xi}, j}$ denotes the $j$-th component of $\Phi_{\bar{\xi}}$, then the vector $\bar{D}_{(i)}$ can be represented in coordinates as follows:

$$
\bar{D}_{(i)}=\sum_{j=1}^{8} D_{\bar{\xi},(i)}\left(\Phi_{\bar{\xi}, j}\right) \frac{\partial}{\partial \bar{e}_{j}} .
$$

Moreover, the function $D_{\bar{\xi},(i)}\left(\Phi_{\bar{\xi} j}\right)$ is a polynomial $P(\bar{e})$ in the variables $\bar{e}=\Phi_{\bar{\xi} j}$, with coefficients independent of $\bar{\xi}$.

In fact, $\bar{D}_{(i)}$ can obviously be written

$$
\bar{D}_{(i)}=\sum_{j=1}^{8} a_{i j} \frac{\partial}{\partial \bar{e}_{j}}
$$

for suitable coefficients $a_{i j}$. On the other hand, if $f=\Phi_{\bar{\xi}}$, then $f_{\bar{\xi}}=$ identity. Hence

$$
D_{\bar{\xi},(i)}\left(\Phi_{\bar{\xi}, m}\right)=\bar{D}_{(i)}\left(\bar{e}_{m}\right)=\sum_{j=1}^{8} a_{i j} \frac{\partial \bar{e}_{m}}{\partial \bar{e}_{j}}=a_{i m} .
$$

Substituting in (3.14), we get the first assertion. Let us compute $D_{\bar{\xi},(i)}\left(\Phi_{\bar{\xi}}\right)$. At a point $\zeta$ we have

$$
D_{\bar{\xi},(i)} \Phi_{\bar{\xi}}(\zeta)=\lim _{s \rightarrow 0} \frac{\Phi_{\bar{\xi}}\left(\exp \left(s D_{\bar{\xi},(i)}\right)(\zeta)\right)-\Phi_{\bar{\xi}}(\zeta)}{s} .
$$

If we call $\bar{e}$ the canonical coordinates of $\zeta$ with respect to $D_{\bar{\xi}}$, the first term in the right hand side of (3.15) becomes

$$
\Phi_{\bar{\xi}}\left(\exp \left(s D_{\bar{\xi},(i)}\right)(\zeta)\right)=\Phi_{\bar{\xi}}\left(\exp \left(s D_{\bar{\xi},(i)}\right)\left(\exp \left(\bar{e} D_{\bar{\xi}}\right)(\bar{\xi})\right)\right)
$$

(by the Campbell-Hausdorff formula)

$$
=\Phi_{\bar{\xi}}\left(\exp \left(\eta D_{\bar{\xi}}\right)(\bar{\xi})\right)=\eta(s),
$$

for a polynomial $\eta(s)$ in the variables $s$ and $\bar{e}$. The derivative in (3.15) is the coefficient of $s$ in $\eta(s)$; hence it is a polynomial in the components of $\bar{e}$. For the reader's convenience we compute these derivatives explicitly:

$$
\begin{gathered}
D_{\bar{\xi},(1)}(\bar{e})=\left(1,0,0,-\frac{\bar{e}_{2}}{2}, 0,-\frac{\bar{e}_{3}}{2},-\frac{\bar{e}_{2}^{2}}{12},-\frac{\bar{e}_{4}}{2}-\frac{\bar{e}_{1} \bar{e}_{2}}{12}\right), \\
D_{\bar{\xi},(2)}(\bar{e})=\left(0,1,0, \frac{\bar{e}_{1}}{2},-\frac{\bar{e}_{3}}{2}, 0,-\frac{\bar{e}_{4}}{2}+\frac{\bar{e}_{1} \bar{e}_{2}}{12},-\frac{\bar{e}_{1}^{2}}{12}\right),
\end{gathered}
$$




$$
D_{\bar{\xi},(3)}(\bar{e})=\left(0,0,1,0, \frac{\bar{e}_{2}}{2}, \frac{\bar{e}_{1}}{2}, 0,0\right), \quad D_{\bar{\xi},(4)}(\bar{e})=\left(0,0,0,1,0,0, \frac{\bar{e}_{2}}{2}, \frac{\bar{e}_{1}}{2},\right),
$$

and $D_{\bar{\xi},(i)}\left(\bar{e}_{j}\right)=\delta_{i, j}$, where $i=5, \cdots, 8, j=1, \cdots, 8$, and $\delta$ is the Kroneker function.

Because of the previous remark the operator

$$
\bar{L}=\bar{D}_{(1)}^{2}+\bar{D}_{(2)}^{2}+\bar{D}_{(3)}
$$

is a sum of squares of linear and nilpotent vector fields of class $C^{\infty}$ independent of $\bar{\xi}$. If we denote by $\bar{\Gamma}$ its fundamental solution, and $\bar{d}$ its natural distance, then $\bar{\Gamma}$ is locally equivalent to $\bar{d}^{2-N}$. Moreover,

$$
\Gamma_{\bar{\xi}}(\xi, \zeta)=\frac{1}{h(\bar{\xi})(X b-Y a)(\bar{\xi})} \bar{\Gamma}\left(\Phi_{\bar{\xi}}(\xi), \Phi_{\bar{\xi}}(\zeta)\right)
$$

and

$$
d_{\bar{\xi}}(\xi, \zeta)=\bar{d}\left(\Phi_{\bar{\xi}}(\xi), \Phi_{\bar{\xi}}(\zeta)\right) .
$$

Let us now study the dependence of the canonical coordinates $\Phi_{\bar{\xi}}(\zeta)$ on the variable $\bar{\xi}$, and as a consequence we will deduce the dependence of the fundamental solution $\Gamma_{\bar{\xi}}(\bar{\xi}, \zeta)$ on the same variable.

Lemma 3.4. Let $U$ be defined as in (2.6), and let $\xi, \bar{\xi}$ be fixed in $U$. Then there exists a constant $C_{3}$, independent of $\xi$ and $\bar{\xi}$, such that for every $\zeta \in U$

$$
\bar{d}\left(\Phi_{\bar{\xi}}(\zeta), \Phi_{\xi_{1}}(\zeta)\right) \leq C_{3}\left(d_{\bar{\xi}}(\bar{\xi}, \xi)+d_{\bar{\xi}}^{\alpha / 3}(\bar{\xi}, \xi) d_{\bar{\xi}}^{1-\alpha / 3}(\bar{\xi}, \zeta)\right) .
$$

Precisely, if $w$ denotes the canonical coordinate of $\Phi_{\bar{\xi}}(\zeta)$ around $\Phi_{\xi_{1}}(\zeta)$ with respect to the vector fields $\bar{D}$, then

$$
\left|w_{i}\right| \leq C_{3}\left(d_{\bar{\xi}}^{|(i)|}(\bar{\xi}, \xi)+d_{\bar{\xi}}^{\alpha}(\bar{\xi}, \xi) d_{\bar{\xi}}^{|(i)|-\alpha}(\bar{\xi}, \zeta)\right),
$$

for every $i=1, \cdots, 8$. Moreover if $\xi=\exp (s S)(\bar{\xi})$, then for every $i$ such that $|(i)| \leq 1$, we have $w_{i}=0$. For $|(i)|>1$

$$
\left|w_{i}\right| \leq C_{3}\left(s d^{|(i)|-2}(\zeta, \xi)+s^{(1+\alpha) / 2} d^{2}(\zeta, \xi)\right) .
$$

Proof. By the definition of the canonical coordinates, $w$ can be expressed as follows:

$$
w=\Phi_{\bar{\xi}, \zeta}\left(\Phi_{\bar{\xi}}^{-1} \Phi_{\xi}(\zeta)\right)
$$

The distances are expressed in terms of canonical coordinates

$$
\eta=\Phi_{\xi}(\zeta), \quad \theta=\Phi_{\bar{\xi}}(\xi)
$$

and we have to estimate $w$ in terms of $\eta$ and $\theta$. Since $\Phi_{\bar{\xi}, \zeta}$ is the inverse mapping of the exponential, we have

$$
\exp \left(w D_{\bar{\xi}}\right)(\zeta)=\Phi_{\bar{\xi}}^{-1}\left(\Phi_{\xi}(\zeta)\right)
$$

(by definition of $\eta$ )

$$
=\Phi_{\bar{\xi}}^{-1}(\eta)=\exp \left(\eta D_{\bar{\xi}}\right)(\bar{\xi})
$$


(since $\left.\bar{\xi}=\exp \left(-\theta D_{\bar{\xi}}\right)(\xi)\right)$

$$
\begin{gathered}
=\exp \left(\eta D_{\bar{\xi}}\right) \exp \left(-\theta D_{\bar{\xi}}\right)(\xi) \\
=\exp \left(\eta D_{\bar{\xi}}\right)\left(\exp \left(-\theta D_{\bar{\xi}}\right) \exp \left(-\eta D_{\xi}\right)(\zeta)\right. \\
=\exp \left(\eta D_{\bar{\xi}}\right) \exp \left(-\theta D_{\bar{\xi}}\right) \exp \left(-\eta D_{\bar{\xi}}-\eta\left(D_{\xi}-D_{\bar{\xi}}\right)\right)(\zeta)
\end{gathered}
$$

If we denote by $f_{i}$ and $g_{i}$ the coefficients of $\partial_{t}$ and $\partial_{x_{3}}$ with respect to the vector fields $D_{\bar{\xi},(i)}$, we get

$$
\begin{gathered}
\eta\left(D_{\xi}-D_{\bar{\xi}}\right)=\sum_{i=1}^{8} \eta_{i}\left(P_{\xi}^{3-|(i)|} f_{i}(\zeta)-P_{\bar{\xi}}^{3-|(i)|} f_{i}(\zeta)\right) \partial_{t} \\
+\sum_{i=3}^{8} \frac{\eta_{i}}{h}\left(P_{\bar{\xi}}^{3-|(i)|} g_{i}(\zeta)-P_{\bar{\xi}}^{3-|(i)|} g_{i}(\zeta)\right)\left(D_{\bar{\xi},(3)}-\frac{f_{3}}{f_{8}} D_{\bar{\xi},(8)}\right)
\end{gathered}
$$

(by Remark 2.8)

$$
\begin{gathered}
=\sum_{i=1}^{8} \eta_{i} O\left(\|\theta\|^{\alpha}\left(\|\eta\|^{3-|(i)|}+\|\theta\|^{3-|(i)|}\right)\right)\left(D_{\bar{\xi},(8)}+D_{\bar{\xi},(3)}\right) \\
=O\left(\|\eta\|\|\theta\|^{\alpha+2}+\|\theta\|^{\alpha}\|\eta\|^{3}\right)\left(D_{\bar{\xi},(8)}+D_{\bar{\xi},(3)}\right) .
\end{gathered}
$$

Hence, if $*$ denotes the law induced by the Campbell-Hausdorff formula, we get

$$
\begin{aligned}
& \exp \left(w D_{\bar{\xi}}\right)(\zeta) \\
& \quad=\exp \left(\eta D_{\bar{\xi}} *\left(-\theta D_{\bar{\xi}}\right) *\left(-\eta D_{\bar{\xi}}+O\left(\|\theta\|^{\alpha+3}+\|\theta\|^{\alpha}\|\eta\|^{3}\right)\left(D_{\bar{\xi},(8)}+D_{\bar{\xi},(3)}\right)\right)(\zeta)\right. \\
& \quad=\exp \left(-\theta D_{\bar{\xi}}+\left(\eta_{1} \theta_{2}-\theta_{1} \eta_{2}\right) D_{\bar{\xi},(4)}+\sum_{i=3}^{8} O\left(\|\theta\|^{2}\|\eta\|+\|\theta\|^{\alpha}\|\eta\|^{3-\alpha}\right) D_{\bar{\xi},(i)}\right) .
\end{aligned}
$$

Since the canonical coordinates are unique, this implies that

$$
\begin{gathered}
w_{i}=\theta_{i} \quad \text { for }|i|=1 \\
\left|w_{i}\right|=\left|\theta_{i}\right|+O\left(\left\|\theta||^{|i|-1}|| \eta\right\|+\|\theta\|^{\alpha}\|\eta\|^{|i|-\alpha}\right) \text { for }|i|>1 .
\end{gathered}
$$

Writing this same assertion in terms of the distances, we get the first part of the thesis. If $\xi=\exp (s S)(\bar{\xi})$, then by arguing as before we get

$$
\eta\left(D_{\bar{\xi}}-D_{\xi}\right)=O\left(\left(\|\eta\| s^{\frac{2+\alpha}{2}}+\|\eta\|^{2} s^{\frac{1+\alpha}{2}}\right)\left(D_{\bar{\xi}, 8}+D_{\bar{\xi}, 3}\right)\right)
$$

and consequently

$$
\begin{gathered}
\exp \left(w D_{\bar{\xi}}\right)(\zeta)=\exp \left(-s S+\eta_{2} s D_{\bar{\xi},(5)}+\eta_{1} s D_{\bar{\xi},(6)}\right) \\
=O\left(\left(\|\eta\| s^{\frac{2+\alpha}{2}}+\|\eta\|^{2} s^{\frac{1+\alpha}{2}}\right)\left(D_{\bar{\xi},(8)}+D_{\bar{\xi},(3)}\right)\right) .
\end{gathered}
$$

Then

$$
\left|w_{i}\right|=-s \delta_{i 3}+O\left(\|\eta\| s+\|\eta\|^{2} s^{\frac{1+\alpha}{2}}\right),
$$

where $\delta$ is the Kronecker function. The second assertion of the thesis is proved. 
A Hölder continuous dependence on the variable $\bar{\xi}$ can now be proved also for $\Gamma_{\bar{\xi}}$. Let $C_{1}, C_{2}, C_{3}$ be the constants introduced in Proposition 3.2 and Lemma 3.4 and let $C_{4}$ be a fixed constant such that

$$
C_{4}>1+\frac{2 C_{2} C_{3}}{C_{1}}, \quad C_{4}>1+\frac{\left(2 C_{3}\right)^{3 /(3-\alpha)} C_{2}}{C_{1}} .
$$

Then

Proposition 3.5. Let $U$ be fixed as in (2.6). There is a constant $C_{5}$, independent of $\xi$ and $\bar{\xi}$, such that for every pair of points $\bar{\xi}$ and $\xi$ in $U, \Gamma_{\bar{\xi}}$ and $\Gamma_{\xi}$ satisfy the following estimate:

$$
\left|\Gamma_{\bar{\xi}}(\bar{\xi}, \zeta)-\Gamma_{\xi}(\xi, \zeta)\right| \leq C_{5}\left(\frac{d(\bar{\xi}, \xi)}{d^{N-1}(\bar{\xi}, \zeta)}+\frac{d^{\alpha}(\bar{\xi}, \xi)}{d^{N-2}(\bar{\xi}, \zeta)}\right),
$$

for every $\zeta$ in the set $U_{\bar{\xi}, \xi}=\left\{\zeta \in U: d(\xi, \zeta) \geq C_{4} d(\xi, \bar{\xi})\right\}$. If $\xi=\exp (s S)(\bar{\xi})$, then

$$
\left|\Gamma_{\bar{\xi}}(\bar{\xi}, \zeta)-\Gamma_{\xi}(\xi, \zeta)\right| \leq C_{5}\left(\frac{s}{d^{N}(\bar{\xi}, \zeta)}+\frac{s^{(1+\alpha) / 2}}{d^{N-2}(\bar{\xi}, \zeta)}\right) .
$$

Proof. By (3.16) we have

$$
\left|\Gamma_{\bar{\xi}}(\bar{\xi}, \zeta)-\Gamma_{\xi}(\xi, \zeta)\right|=\left|\frac{\bar{\Gamma}\left(0, \Phi_{\bar{\xi}}(\zeta)\right)}{h(\bar{\xi})(X b-Y a)(\bar{\xi})}-\frac{\bar{\Gamma}\left(0, \Phi_{\xi}(\zeta)\right)}{h(\xi)(X b-Y a)(\xi)}\right|
$$

(by the mean value theorem)

$$
=|w \bar{D} \bar{\Gamma}(0, \theta)|+\frac{d^{\alpha}(\xi, \bar{\xi})}{d^{N-2}(\zeta, \bar{\xi})},
$$

where $w$ are the canonical coordinates of $\Phi_{\bar{\xi}}(\zeta)$ around $\Phi_{\xi}(\zeta)$ with respect to the vector fields $\bar{D}$, and $\theta$ is a suitable point belonging to the integral curve $\exp (\tau w D)\left(\Phi_{\xi}(\zeta)\right)$. Consequently

$$
\begin{gathered}
\bar{d}(\theta, 0) \geq \bar{d}\left(0, \Phi_{\bar{\xi}}(\zeta)\right)-\bar{d}\left(\theta, \Phi_{\bar{\xi}}(\zeta)\right) \\
\quad \geq \bar{d}\left(0, \Phi_{\bar{\xi}}(\zeta)\right)-\bar{d}\left(\Phi_{\xi}(\zeta), \Phi_{\bar{\xi}}(\zeta)\right)
\end{gathered}
$$

(by (3.16) and Lemma 3.4)

$$
\geq d_{\bar{\xi}}(\bar{\xi}, \zeta)-C_{3} d_{\bar{\xi}}(\bar{\xi}, \xi)-C_{3} d_{\bar{\xi}}^{\alpha / 3}(\bar{\xi}, \zeta) d_{\bar{\xi}}^{1-\alpha / 3}(\bar{\xi}, \xi)
$$

Note that

$$
\begin{gathered}
\left.d_{\bar{\xi}}(\bar{\xi}, \zeta) \geq \quad \text { (by Proposition } 3.2\right) C_{1} d(\bar{\xi}, \zeta) \\
\left.\geq C_{1}(d(\xi, \zeta)-d(\xi, \bar{\xi})) \geq \quad \text { if } \zeta \in D\right) C_{1}\left(C_{4}-1\right) d(\xi, \bar{\xi}) \\
\geq \quad \text { (by Proposition 3.2) } \frac{C_{1}\left(C_{4}-1\right)}{C_{2}} d_{\bar{\xi}}(\xi, \bar{\xi}) .
\end{gathered}
$$

Then by (3.19)

$$
\begin{gathered}
\bar{d}(\theta, 0) \geq d_{\bar{\xi}}(\bar{\xi}, \zeta)-C_{3} d_{\bar{\xi}}(\bar{\xi}, \xi)-C_{3} d_{\bar{\xi}}^{\alpha / 3}(\bar{\xi}, \zeta) d_{\bar{\xi}}^{1-\alpha / 3}(\bar{\xi}, \xi) \\
\geq \frac{1}{2}\left(d_{\bar{\xi}}(\bar{\xi}, \zeta)-2 C_{3} d_{\bar{\xi}}(\bar{\xi}, \xi)\right)+\frac{1}{2}\left(d_{\bar{\xi}}^{1-\alpha / 3}(\bar{\xi}, \zeta)-2 C_{3} d_{\bar{\xi}}^{1-\alpha / 3}(\bar{\xi}, \xi)\right) d_{\bar{\xi}}^{\alpha / 3}
\end{gathered}
$$




$$
\begin{aligned}
& \geq \frac{1}{2} d_{\bar{\xi}}(\bar{\xi}, \zeta)(1\left.-\frac{2 C_{3} C_{2}}{C_{1}\left(C_{4}-1\right)}\right)+\frac{1}{2} d_{\bar{\xi}}(\bar{\xi}, \zeta)\left(1-2 C_{3}\left(\frac{C_{2}}{C_{1}\left(C_{4}-1\right)}\right)^{1-\alpha / 3}\right) \\
& \geq\left(\text { for the choice of } C_{4}\right) \frac{1}{2} d_{\bar{\xi}}(\bar{\xi}, \zeta) .
\end{aligned}
$$

By the standard estimates of $\bar{\Gamma}$ in the directions of the vector fields we get

$$
\left|\bar{D}_{\sigma} \bar{\Gamma}(0, \theta)\right| \leq C d_{\bar{\xi}}^{-N+2-|\sigma|}(\bar{\xi}, \zeta) .
$$

By Lemma 3.4 we conclude that

$$
|\omega \bar{D} \bar{\Gamma}(0, \theta)| \leq C\left(\frac{d(\bar{\xi}, \xi)}{d^{N-1}(\bar{\xi}, \zeta)}+\frac{d^{\alpha}(\bar{\xi}, \xi)}{d^{N-2}(\bar{\xi}, \zeta)}\right) .
$$

If $\xi=\exp (s S)(\bar{\xi})$, then by (3.18), (3.20) and Lemma 3.4 we conclude the second part of the thesis.

Finally, we recall a property of the Taylor polynomial in the frozen directions:

Lemma 3.6. Let $f \in C_{L}^{m, \alpha}$ and $m \leq 3$, and let $P_{\bar{\xi}}^{m} f$ be the polynomial

$$
P_{\bar{\xi}}^{m} f(\xi)=\sum_{i=1}^{m} \frac{1}{i !} \sum_{l e n g t h(\sigma)=i,|(\sigma)| \leq m} \bar{e}_{\sigma} D_{\sigma} f(\bar{\xi})
$$

where $\bar{e}$ are the frozen canonical coordinates. Then

$$
f(\xi)=P_{\bar{\xi}}^{m} f(\xi)+O\left(d^{m+\alpha}(\xi, \bar{\xi})\right)
$$

and

$$
\left|P_{\xi}^{m} f(\zeta)-P_{\bar{\xi}}^{m} f(\zeta)\right| \leq C d^{\alpha}(\xi, \bar{\xi})\left(d^{m}(\xi, \bar{\xi})+d^{m}(\bar{\xi}, \zeta)\right) .
$$

Besides, if $f \in C_{L}^{m+1, \alpha}$ and $\xi=\exp (s S)(\bar{\xi})$, then

$$
\left|P_{\xi}^{m} f(\zeta)-P_{\bar{\xi}}^{m} f(\zeta)\right| \leq C s^{(1+\alpha) / 2}\left(d^{m}(\xi, \bar{\xi})+d^{m}(\bar{\xi}, \zeta)\right) .
$$

Proof. Let us fix two points $\xi$ and $\bar{\xi}$ and denote $\bar{e}=\Phi_{\bar{\xi}}(\xi)$. Then, by using (3.4), (3.5), (3.11), 3.9), (3.10), (3.12), (3.13), it is easy to recognize that for every $i=1, \cdots, 8$

$$
\bar{e}_{3}=e_{i}+O\left(d^{3+\alpha}(\xi, \bar{\xi})\right)
$$

Hence, by Proposition 2.7 the assertion is true for $m=1,2$. Let us prove the assertion for $m=3$. If $f \in C_{L}^{m, \alpha}(U)$ then $f \in C_{\text {euclid }}^{1}$ in the Euclidean sense, so that, if

$$
\gamma(s)=\exp (s e D)(\bar{\xi})
$$

we have

$$
f(\xi)=f(\bar{\xi})+\sum_{i=1}^{8} e_{i} \int_{0}^{1} D_{(i)} f(\gamma(s)) d s
$$

(by induction) 


$$
\begin{aligned}
=f(\bar{\xi})+ & \sum_{i=1}^{2} e_{i} \int_{0}^{1} P_{\bar{\xi}}^{2}\left(D_{(i)} f(\gamma(s))\right) d s+\sum_{i=3}^{4} e_{i} \int_{0}^{1} P_{\bar{\xi}}^{1}\left(D_{(i)} f(\gamma(s))\right) d s \\
& +\sum_{i=5}^{8} e_{i} \int_{0}^{1} D_{(i)} f(\gamma(s)) d s+O\left(d^{3+\alpha}(\xi, \bar{\xi})\right)
\end{aligned}
$$

(since the canonical coordinate of the point $\gamma(s)$ is $s e$ )

$$
\begin{array}{r}
=f(\bar{\xi})+\sum_{i=1}^{2} e_{i} \int_{0}^{1}\left(D_{(i)} f(\bar{\xi})+\sum_{j=1}^{4} e_{j} s D_{(j)} D_{(i)} f(\bar{\xi})\right. \\
\left.+\frac{1}{2} \sum_{m, j=1}^{2} s^{2} e_{m} e_{j} D_{(m)} D_{(j)} D_{(i)} f(\bar{\xi})\right) d s \\
+\sum_{i=3}^{4} e_{i} \int_{0}^{1}\left(D_{(i)} f(\bar{\xi})+\sum_{j=1}^{2} e_{j} s D_{(j)} D_{(i)} f(\bar{\xi}) d s\right) \\
+\sum_{i=5}^{8} e_{i} \int_{0}^{1} D_{(i)} f(\bar{\xi}) d s+O\left(d^{3+\alpha}(\xi, \bar{\xi})\right)
\end{array}
$$

(computing the integration and reordering all terms)

$$
=\sum_{i=1}^{m} \frac{1}{i !} \sum_{l e n g t h(\sigma)=i,|(\sigma)| \leq m} e_{\sigma} D_{\sigma} f(\bar{\xi})=P_{\bar{\xi}}^{m} f(\xi),
$$

by (3.21). Since we have already computed all the derivatives $D_{\bar{\xi},(i)} \bar{e}$ in Remark 3.3, a direct computation proves that for every $i=1, \cdots, 8$

$$
D_{\bar{\xi},(i)} P_{\bar{\xi}}^{3}(f)=P_{\bar{\xi}}^{3-|(i)|}\left(D_{\bar{\xi},(i)} f\right) .
$$

Using this fact, the proof of the second assertion can be performed as in the second part of Proposition 2.7 starting from identity (2.12).

\section{Regularity Results For the linear operator}

Our regularity result will be achieved by a technique introduced in CM2]. In order to obtain the regularity of solutions of the linear equation with low regular coefficients we follow the formulation of this method provided in [CPP]. It is based on the following definition and lemma.

Definition 4.1. Let $\left(F_{\varepsilon}\right)$ be a family of continuous functions on $U \times U$ and let $f: U \rightarrow \mathbb{R}$. We say that

$$
F_{\varepsilon}(\xi ; \bar{\xi}) \rightarrow f(\bar{\xi}) \quad \text { as } \varepsilon \rightarrow 0
$$

locally uniformly of order $m+\alpha$ if for every compact set $K \subset \subset U$ there exist $C>0$, $\nu>0$ such that $\forall \xi, \bar{\xi} \in K, d_{\bar{\xi}}(\xi, \bar{\xi}) \leq \nu \varepsilon$,

$$
\left|F_{\varepsilon}(\xi, \bar{\xi})-f(\bar{\xi})\right| \leq C \varepsilon^{m+\alpha} .
$$


Lemma 4.2. Let $|\sigma| \geq 1$ and let $u \in C_{L}^{|\sigma|-1, \alpha}(U)$ be a function which can represented as

$$
u(\xi)=I_{1}(\xi, \bar{\xi})+I_{2}(\xi, \bar{\xi}),
$$

where $I_{1}$ is of class $C_{L}^{|\sigma|, \alpha}(U)$ as a function of $\xi$ in $U$. Let $D_{\sigma} I_{1}(\xi, \bar{\xi})$ denote the derivative with respect to the first variable, and assume that the function $\xi \rightarrow$ $D_{\sigma} I_{1}(\xi, \bar{\xi})$ is continuous in $\xi$ uniformly in $\bar{\xi}$. Assume that there exist a family $I_{2, \varepsilon}$ of smooth functions and a continuous function $I_{2}^{\sigma}$ such that

$$
\begin{gathered}
I_{2, \varepsilon}(\xi, \bar{\xi}) \rightarrow I_{2}(\bar{\xi}, \bar{\xi}) \quad \text { of } \text { order }|\sigma|+\alpha, \\
D_{\sigma} I_{2, \varepsilon}(\xi, \bar{\xi}) \rightarrow I_{2}^{\sigma}(\bar{\xi}) \quad \text { of order } \alpha .
\end{gathered}
$$

Then $u \in C_{L}^{|\sigma|}(U)$, and for every $\bar{\xi}$ in $U$

$$
D_{\sigma} u(\bar{\xi})=D_{\sigma} I_{1}(\bar{\xi}, \bar{\xi})+I_{2}^{\sigma}(\bar{\xi}) .
$$

Using this lemma, we will be able to obtain the regularity of solutions.

4.1. Representation formulas. In order to apply Lemma 4.2, we need a representation formula for solutions of class $u \in C_{L, l o c}^{3, \alpha}(\Omega \times]-1,1[)$ of (1.12). This will be obtained by using the fundamental solution $\Gamma_{\bar{\xi}}$ as a parametrix of the fundamental solution of $L$.

We can extend $u$, and all the coefficients of the operator on $\Omega \times]-1,1\left[\times R^{4}\right.$, as constant functions of the variables $\left(x_{4}, \cdots, x_{7}\right)$. For a fixed $\xi_{0}$ we can obviously study the regularity of $u$ on the open set $U$ defined in (2.6). We then fix $U \subset \subset$ $U_{1} \subset \subset \Omega \times R^{5}$, and we choose a function $\phi \in C_{0}^{\infty}\left(\Omega \times R^{5}\right)$ such that $\phi \equiv 1$ on $U_{1}$, so that $v_{\mid U}=(v \phi)_{\mid U}$.

Theorem 4.3. Let $3 \leq m \leq 7$. Assume that the coefficients $a, b, h$ in equation (1.12) are of class $C_{\text {euclid }}^{2, \alpha}(U) \cap C_{L}^{m-1, \alpha}(U), c, g \in C_{L}^{m-2, \alpha}(U)$ and that $\partial_{t} a \in$ $C_{L}^{\alpha}(U), \partial_{t} b \in C_{L}^{\alpha}(U)$. Let $u \in C_{L}^{m-1, \alpha}$ be a classical solution of (1.12) such that $\partial_{t} u \in C_{L}^{1, \alpha}(U), S u \in C_{L}^{1, \alpha}(U)$. For every $\bar{\xi} \in U_{2}, u=u \phi$ can be represented as

$$
+\sum_{|\rho|,|\theta| \leq 1} \int e_{\rho} D_{\bar{\xi}, \theta} \Gamma_{\bar{\xi}}(\xi, \zeta) N_{2, m, \theta, \rho}(\zeta, \bar{\xi}) d \zeta+\sum_{|\rho| \leq 1} \int D_{\bar{\xi}, \rho} \Gamma_{\bar{\xi}}(\xi, \zeta) N_{3, m, \rho}(\zeta, \bar{\xi}) d \zeta,
$$

where $N_{i, m, \rho}(\cdot, \bar{\xi})$ is supported in the support of $\phi$, and

(i) $\operatorname{supp}\left(N_{1}(\cdot, \bar{\xi})\right) \subset \operatorname{supp}(\nabla \phi)$, and

$$
\left|N_{1}(\zeta, \xi)-N_{1}(\zeta, \bar{\xi})\right| \leq C d_{\bar{\xi}}(\bar{\xi}, \xi)^{\alpha} ;
$$

(ii) $N_{2, m, \theta, \rho}(\cdot, \bar{\xi})$ is of class $C_{L}^{m-|\rho|+|\theta|-1, \alpha}$, and, for every multi-index $\sigma$ such that $|\sigma| \leq m-|\rho|+|\theta|-1$,

$$
\left|D_{\sigma} N_{2, m, \theta, \rho}(\zeta, \bar{\xi})-D_{\sigma} N_{2, m, \theta, \rho}(\zeta, \xi)\right| \leq C d_{\bar{\xi}}(\bar{\xi}, \xi)^{\alpha}, \quad \forall \xi, \bar{\xi} \in U_{2},
$$

where the derivative is with respect to the first variable;

(iii) there exists a constant $C$, only dependent on the choice of $\phi$ and $U_{1}$, such that

$$
\left|N_{3, m, \rho}(\zeta, \bar{\xi})\right| \leq C d_{\bar{\xi}}^{m-2+\alpha+|\rho|}(\bar{\xi}, \zeta)
$$

and 


$$
\left|N_{3, m, \rho}(\zeta, \xi)-N_{3, m, \rho}(\zeta, \bar{\xi})\right| \leq C d_{\bar{\xi}}(\bar{\xi}, \xi)^{\alpha}\left(d_{\bar{\xi}}(\bar{\xi}, \zeta)^{m-2+|\rho|}+d_{\xi}(\xi, \zeta)^{m-2+|\rho|}\right),
$$

for every $\xi, \bar{\xi} \in U_{2}$.

Proof. If $m \leq 4$ we set $i=3, j=4$, and if $m \geq 5$ we set $i=j=m-1$. In this way we have in any case

$$
S u \in C_{L}^{i-2, \alpha}(U), \quad \partial_{t} u \in C_{L}^{j-3, \alpha}(U) .
$$

By the definition of fundamental solution we have

$$
u \phi(\xi)=\int \Gamma_{\bar{\xi}}(\xi, \zeta) L_{\bar{\xi}}(u \phi)(\zeta) d \zeta
$$

Since $u$ is a solution of equation (11.12), we get

$$
\begin{gathered}
L_{\bar{\xi}}(u \phi)=u(\zeta) L_{\bar{\xi}} \phi(\zeta)+2 X_{\bar{\xi}} u(\zeta) X_{\bar{\xi}} \phi(\zeta)+2 Y_{\bar{\xi}} u(\zeta) Y_{\bar{\xi}} \phi(\zeta) \\
+\left(L_{\bar{\xi}} u-L u+g\right) \phi(\zeta)
\end{gathered}
$$

(with a direct computation - see for example [C], where the computation is carried out in detail)

$$
\begin{gathered}
=\left(u(\zeta) L_{\bar{\xi}} \phi(\zeta)+2 X_{\bar{\xi}} u(\zeta) X_{\bar{\xi}} \phi(\zeta)+2 Y_{\bar{\xi}} u(\zeta) Y_{\bar{\xi}} \phi(\zeta)\right) \\
-\left(a-P_{\bar{\xi}}^{2} a\right) X \partial_{t} u \phi(\zeta)-\left(a-P_{\bar{\xi}}^{2} a\right) X_{\bar{\xi}} \partial_{t} u \phi(\zeta) \\
-\left(b-P_{\bar{\xi}}^{2} b\right) Y \partial_{t} u \phi(\zeta)-\left(b-P_{\bar{\xi}}^{2} b\right) Y_{\bar{\xi}} \partial_{t} u \phi(\zeta)-\frac{\left(h-P_{\bar{\xi}}^{1} h\right)}{h} S u \phi(\zeta) \\
-\frac{\left(h-P_{\bar{\xi}}^{1} h\right)}{h}(X a+Y b-c) \partial_{t} u \phi(\zeta)+g \phi(\zeta) .
\end{gathered}
$$

Integrating each term separately, and calling the resulting terms $A_{0}, \cdots, A_{7}$, we get

$$
u \phi(\xi)=\sum_{i=0}^{7} A_{i}(\xi, \bar{\xi}) .
$$

Now we can study each of them separately. First,

$$
A_{0}(\xi, \bar{\xi})=\int \Gamma_{\bar{\xi}}(\xi, \zeta)\left(u(\zeta) L_{\bar{\xi}} \phi(\zeta)+2 X_{\bar{\xi}} u(\zeta) X_{\bar{\xi}} \phi(\zeta)+2 Y_{\bar{\xi}} u(\zeta) Y_{\bar{\xi}} \phi(\zeta)\right)
$$

is of type $N_{1}$. Next,

$$
\begin{gathered}
A_{1}(\xi, \bar{\xi})=-\int \Gamma_{\bar{\xi}}(\xi, \zeta)\left(a-P_{\bar{\xi}}^{2} a\right)(\zeta) X \partial_{t} u(\zeta) \phi(\zeta) d \zeta \\
=-\int \Gamma_{\bar{\xi}}(\xi, \zeta)\left(a-P_{\bar{\xi}}^{2} a\right)(\zeta)\left(X \partial_{t} u(\zeta)-P_{\bar{\xi}}^{j-4}\left(X \partial_{t} u\right)(\zeta)\right) \phi(\zeta) d \zeta \\
-\int \Gamma_{\bar{\xi}}(\xi, \zeta)\left(a-P_{\bar{\xi}}^{2} a\right)(\zeta) P_{\bar{\xi}}^{j-4}\left(X \partial_{t} u\right)(\zeta) \phi(\zeta) d \zeta
\end{gathered}
$$

By the properties of the fundamental solution, and of the Taylor polynomials proved in Lemma 3.6 we have

$$
\left|a-P_{\bar{\xi}}^{2} a\right|\left|X \partial_{t} u-P_{\bar{\xi}}^{j-4}\left(X \partial_{t} u\right)\right| \leq d_{\bar{\xi}}^{m-2+\alpha}(\bar{\xi}, \zeta) .
$$


Besides,

$$
\begin{gathered}
\left|\left(a-P_{\bar{\xi}}^{2} a\right)\left(X \partial_{t} u-P_{\bar{\xi}}^{j-4}\left(X \partial_{t} u\right)\right)-\left(a-P_{\xi}^{2} a\right)\left(X \partial_{t} u-P_{\xi}^{j-4}\left(X \partial_{t} u\right)\right)\right| \\
\leq\left|P_{\xi}^{2} a-P_{\bar{\xi}}^{2} a\right|\left|X \partial_{t} u-P_{\bar{\xi}}^{j-4}\left(X \partial_{t} u\right)\right|+\left|a-P_{\xi}^{2} a\right|\left|P_{\bar{\xi}}^{j-4}\left(X \partial_{t} u\right)-P_{\xi}^{j-4}\left(X \partial_{t} u\right)\right| \\
\leq C d_{\bar{\xi}}(\bar{\xi}, \xi)^{\alpha}\left(d_{\bar{\xi}}(\bar{\xi}, \zeta)^{m-2}+d_{\xi}(\xi, \zeta)^{m-2}\right) .
\end{gathered}
$$

Hence the kernel in the first integral is of type $N_{3, m, 0}$, while the kernel in the second integral is of type $N_{2, m, 0,0}$, always by Lemma 3.6.

Let us now integrate the second term on the right hand side of (4.5):

$$
A_{2}(\xi, \bar{\xi})=-\int \Gamma_{\bar{\xi}}(\xi, \zeta)\left(a-P_{\bar{\xi}}^{2} a\right) X_{\bar{\xi}} \partial_{t} u \phi(\zeta) d \zeta
$$

(integrating by parts)

$$
\begin{aligned}
& =\int X_{\bar{\xi}} \Gamma_{\bar{\xi}}(\xi, \zeta)\left(a-P_{\bar{\xi}}^{2} a\right)\left(\partial_{t} u-\partial_{t} u(\bar{\xi})\right) \phi(\zeta) d \zeta \\
& +\int \Gamma_{\bar{\xi}}(\xi, \zeta)\left(X_{\bar{\xi}} a-X_{\bar{\xi}} P_{\bar{\xi}}^{2} a\right)\left(\partial_{t} u-\partial_{t} u(\bar{\xi})\right) \phi(\zeta) d \zeta \\
& +\int \Gamma_{\bar{\xi}}(\xi, \zeta)\left(a-P_{\bar{\xi}}^{2} a\right)\left(\partial_{t} u-\partial_{t} u(\bar{\xi})\right) X_{\bar{\xi}} \phi(\zeta) d \zeta
\end{aligned}
$$

(since $X_{\bar{\xi}}=X-\left(a-P_{\bar{\xi}}^{2} a\right) \partial_{t}$, we can change the second term as follows)

$$
\begin{aligned}
& =\int X_{\bar{\xi}} \Gamma_{\bar{\xi}}(\xi, \zeta)\left(a-P_{\bar{\xi}}^{2} a\right)\left(\partial_{t} u-\partial_{t} u(\bar{\xi})\right) \phi(\zeta) d \zeta \\
& +\int \Gamma_{\bar{\xi}}(\xi, \zeta)\left(X a-P_{\bar{\xi}}^{1}(X a)\right)\left(\partial_{t} u-\partial_{t} u(\bar{\xi})\right) \phi(\zeta) d \zeta \\
& -\int \Gamma_{\bar{\xi}}(\xi, \zeta)\left(a-P_{\bar{\xi}}^{2} a\right) \partial_{t} a(\zeta)\left(\partial_{t} u-\partial_{t} u(\bar{\xi})\right) \phi(\zeta) d \zeta \\
& +\int \Gamma_{\bar{\xi}}(\xi, \zeta)\left(a-P_{\bar{\xi}}^{2} a\right)\left(\partial_{t} u-\partial_{t} u(\bar{\xi})\right) X_{\bar{\xi}} \phi(\zeta) d \zeta
\end{aligned}
$$

(now we add and subtract some term in each of the previous integrals, in order to use their Taylor development. For simplicity we put $P_{\bar{\xi}}^{0} a=0$ )

$$
\begin{aligned}
& =\int X_{\bar{\xi}} \Gamma_{\bar{\xi}}(\xi, \zeta)\left(a-P_{\bar{\xi}}^{2} a\right)\left(\partial_{t} u-P_{\bar{\xi}}^{j-3}\left(\partial_{t} u\right)\right) \phi(\zeta) d \zeta \\
+ & \int X_{\bar{\xi}} \Gamma_{\bar{\xi}}(\xi, \zeta)\left(a-P_{\bar{\xi}}^{2} a\right)\left(P_{\bar{\xi}}^{j-3}\left(\partial_{t} u\right)-\partial_{t} u(\bar{\xi})\right) \phi(\zeta) d \zeta \\
& +\int \Gamma_{\bar{\xi}}(\xi, \zeta)\left(X a-P_{\bar{\xi}}^{1}(X a)\right)\left(\partial_{t} u-P_{\bar{\xi}}^{j-3}\left(\partial_{t} u\right)\right) \phi(\zeta) d \zeta \\
+ & \int \Gamma_{\bar{\xi}}(\xi, \zeta)\left(X a-P_{\bar{\xi}}^{1}(X a)\right)\left(P_{\bar{\xi}}^{j-3}\left(\partial_{t} u\right)-\partial_{t} u(\bar{\xi})\right) \phi(\zeta) d \zeta \\
& -\int \Gamma_{\bar{\xi}}(\xi, \zeta)\left(a-P_{\bar{\xi}}^{2} a\right) \partial_{t} a(\zeta)\left(\partial_{t} u-P_{\bar{\xi}}^{j-3}\left(\partial_{t} u\right)\right) \phi(\zeta) d \zeta
\end{aligned}
$$




$$
\begin{gathered}
-\int \Gamma_{\bar{\xi}}(\xi, \zeta)\left(a-P_{\bar{\xi}}^{2} a\right)\left(\partial_{t} a(\zeta)-P_{\bar{\xi}}^{m-4}\left(\partial_{t} a\right)\right)\left(P_{\bar{\xi}}^{j-3} \partial_{t} u-\partial_{t} u(\bar{\xi})\right) \phi(\zeta) d \zeta \\
-\int \Gamma_{\bar{\xi}}(\xi, \zeta)\left(a-P_{\bar{\xi}}^{2} a\right) P_{\bar{\xi}}^{m-4} \partial_{t} a(\zeta)\left(P_{\bar{\xi}}^{j-3} \partial_{t} u-\partial_{t} u(\bar{\xi})\right) \phi(\zeta) d \zeta \\
+\int \Gamma_{\bar{\xi}}(\xi, \zeta)\left(a-P_{\bar{\xi}}^{2} a\right)\left(\partial_{t} u-\partial_{t} u(\bar{\xi})\right) X_{\bar{\xi}} \phi(\zeta) d \zeta
\end{gathered}
$$

The kernel in the first integral is of type $N_{3, m, 1}$, the second one is of type $N_{2, m, 1,1}$, the kernels in integrals $3,5,6$ are of type $N_{3, m, 0}$, the kernel in the fourth integral is of type $N_{2, m, 0,1}$, the one in the seventh integral is of type $N_{2, m, 0,0}$ and the one in the eighth is of type $N_{1}$.

The third term in (4.5) can be handled exactly as $A_{1}(\xi, \bar{\xi})$, while the fourth has the same behavior as $A_{2}(\xi, \bar{\xi})$. Let us consider $A_{5}$, and denote $m=\min (3, i-2)$ :

$$
\begin{aligned}
A_{5}(\xi, \bar{\xi}) & =-\int \Gamma_{\bar{\xi}}(\xi, \zeta)\left(1-\frac{P_{\bar{\xi}}^{1} h}{h}\right)\left(S u-P_{\bar{\xi}}^{m}(S u)\right) \phi(\zeta) d \zeta \\
& -\int \Gamma_{\bar{\xi}}(\xi, \zeta)\left(1-\frac{P_{\bar{\xi}}^{1} h}{h}\right) P_{\bar{\xi}}^{m}(S u) \phi(\zeta) d \zeta .
\end{aligned}
$$

Here the first term has the behavior of $N_{3, m, 0}$, while the second is of type $N_{2, m, 0,0}$. Next,

$$
\begin{gathered}
A_{6}(\xi, \bar{\xi})=-\int \Gamma_{\bar{\xi}}(\xi, \zeta)\left(1-\frac{P_{\bar{\xi}}^{1} h}{h}\right)(X a+Y b-c) \partial_{t} u \phi(\zeta) d \zeta \\
-\int \Gamma_{\bar{\xi}}(\xi, \zeta)\left(1-\frac{P_{\bar{\xi}}^{\frac{1}{\xi}}}{h}\right)(X a+Y b-c)\left(\partial_{t} u-P_{\bar{\xi}}^{j-3} \partial_{t} u\right) \phi(\zeta) d \zeta \\
-\int \Gamma_{\bar{\xi}}(\xi, \zeta)\left(1-\frac{P_{\bar{\xi}}^{1} h}{h}\right)\left((X a+Y b-c)-P_{\bar{\xi}}^{m-2}(X a+Y b-c)\right) P_{\bar{\xi}}^{j-3} \partial_{t} u \phi(\zeta) d \zeta \\
-\int \Gamma_{\bar{\xi}}(\xi, \zeta)\left(1-\frac{P_{\bar{\xi}}^{1} h}{h}\right) P_{\bar{\xi}}^{m-2}(X a+Y b) P_{\bar{\xi}}^{j-3} \partial_{t} u \phi(\zeta) d \zeta
\end{gathered}
$$

The kernels in the first two integrals are of type $N_{3, m, 0}$, while the last one is of type $N_{2, m, 0,0}$. Finally,

$$
A_{7}(\xi, \bar{\xi})=\int \Gamma_{\bar{\xi}}(\xi, \zeta) g \phi(\zeta) d \zeta
$$

is of type $N_{2, m, 0,0}$.

We will need also another representation of $u$ :

Theorem 4.4. Let $3 \leq m \leq 7$. Assume that the coefficients $a, b, h$ in equation (1.12) are of class $C_{L}^{m-1, \alpha}(\bar{U}), c, g \in C_{L}^{m-2, \alpha}(U)$, and that $\partial_{t} a \in C_{L}^{\alpha}(U), \partial_{t} b \in$ 
$C_{L}^{\alpha}(U)$. Let $u \in C_{L}^{m-1, \alpha}$ be a classical solution of (1.12) such that $\partial_{t} u \in C_{L}^{1, \alpha}(U)$, $S u \in C_{L}^{1, \alpha}(U)$. For every $\bar{\xi} \in U_{2}, u=u \phi$ can be represented as

$$
\begin{gathered}
u(\xi)=u \phi(\xi)=\int \Gamma_{\bar{\xi}}(\zeta, \xi) N_{1}(\zeta, \bar{\xi}) d \zeta \\
+\sum_{|\rho|,|\theta| \leq 1} \int e_{\rho} D_{\bar{\xi}, \theta} \Gamma_{\bar{\xi}}(\xi, \zeta) \widetilde{N}_{2, m, \theta, \rho}(\zeta, \bar{\xi}) d \zeta+\sum_{|\rho| \leq 1} \int D_{\bar{\xi}, \rho} \Gamma_{\bar{\xi}}(\xi, \zeta) \widetilde{N}_{3, m, \rho}(\zeta, \bar{\xi}) d \zeta
\end{gathered}
$$

where $\widetilde{N}_{i, m, \rho}(\cdot, \bar{\xi})$ is supported in the same set as $\phi$, and

(i) $\operatorname{supp}\left(N_{1}(\cdot, \bar{\xi})\right) \subset \operatorname{supp}(\nabla \phi)$ and, if $\xi=\exp (s S)(\bar{\xi})$, then

$$
\left|N_{1}(\zeta, \xi)-N_{1}(\zeta, \bar{\xi})\right| \leq C s^{(1+\alpha) / 2}
$$

(ii) $\widetilde{N}_{2, m, \theta, \rho}(\cdot, \bar{\xi})$ is of class $C_{L}^{m-|\rho|+|\theta|-2, \alpha}$, and, for every multi-index $\sigma$ such that $|\sigma| \leq m-|\rho|+|\theta|-2$, for every $\xi=\exp (s S)(\bar{\xi})$,

$$
\left|D_{\sigma} \widetilde{N}_{2, m-1, \theta, \rho}\left(\xi_{1}, \bar{\xi}\right)-D_{\sigma} \widetilde{N}_{2, m-1, \theta, \rho}\left(\xi_{1}, \xi\right)\right| \leq C s^{(1+\alpha) / 2},
$$

where the derivative is taken with respect to the first variable;

(iii) there exists a constant $C$, only dependent on the choice of $\phi$ and $K_{1}$, such that

$$
\left|\widetilde{N}_{3, m, \rho}(\zeta, \bar{\xi})\right| \leq C d_{\bar{\xi}}^{m-3+\alpha}(\bar{\xi}, \zeta)
$$

and

$$
\begin{aligned}
& \left|\widetilde{N}_{3, m-1, \rho}(\zeta, \bar{\xi})-\widetilde{N}_{3, m-1, \rho}(\zeta, \xi)\right| \leq C s^{(\alpha+1) / 2}\left(d_{\bar{\xi}}(\bar{\xi}, \zeta)^{m-3+\alpha}+d_{\xi}(\xi, \zeta)^{m-3+\alpha}\right), \\
& \text { if } \xi=\exp (s S)(\bar{\xi})
\end{aligned}
$$

Proof. Let us represent the function $u$ as in (4.6), with the same notations as in the preceding theorem. The function $A_{1}$ can also be expressed as follows:

$$
\begin{aligned}
A_{1}(\xi, \bar{\xi})= & -\int \Gamma_{\bar{\xi}}(\xi, \zeta)\left(a-P_{\bar{\xi}}^{2} a\right)(\zeta)\left(X \partial_{t} u(\zeta)-P_{\bar{\xi}}^{j-5}\left(X \partial_{t} u\right)(\zeta)\right) \phi(\zeta) d \zeta \\
& -\int \Gamma_{\bar{\xi}}(\xi, \zeta)\left(a-P_{\bar{\xi}}^{2} a\right)(\zeta) P_{\bar{\xi}}^{j-5}\left(X \partial_{t} u\right)(\zeta) \phi(\zeta) d \zeta
\end{aligned}
$$

Since $X \partial_{t} u$ is of class $C_{L}^{j-4, \alpha}$ and we consider the Taylor polynomial of order $j-5$, then these two terms are of type $\widetilde{N}_{3, m, 0}, \widetilde{N}_{2, m, 0,0}$ respectively.

Analogously, if we represent $A_{2}$ as before, but using Taylor developments of $\partial_{t} u$ of order $j-4$, it turns out to be the sum of 8 terms of the following type. The first kernel is of type $\widetilde{N}_{3, m, 1}$, the second of type $\widetilde{N}_{2, m, 1,1}$ kernels $3,5,6$, of type $\widetilde{N}_{3, m, 0}$, kernel 4 of type $\widetilde{N}_{2, m, 0,1}$, kernel 7 of type $\widetilde{N}_{2, m, 0,0}$ and kernel 8 of type $N_{1}$.

Using the Taylor expansion of $S u$ of order $i-3$, we represent $A_{5}$ as a sum of terms of type $\widetilde{N}_{3, m, 0}$ and $\widetilde{N}_{2, m, 0,0}$ respectively. 


\subsection{Low order regularity.}

Theorem 4.5. Let $3 \leq m \leq 7$. Let us assume that the coefficients $a, b, h$ in equation (1.12) are of class $C_{\text {euclid }}^{2, \alpha}(U) \cap C_{L}^{m-1, \alpha}(U), c \in C_{\text {euclid }}^{2}(U) \cap C_{L}^{m-2, \alpha}(U)$, $g \in C_{L}^{m-2, \alpha}(U)$ and $\partial_{t} a \in C_{L}^{\alpha}(U), \partial_{t} b \in C_{L}^{\alpha}(U)$. Let $u \in C_{L}^{m-1, \alpha}$ be a classical solution of (1.12) such that $\partial_{t} u \in C_{L}^{1, \alpha}(U), S u \in C_{L}^{1, \alpha}(U)$. Then $u \in C_{L}^{m, \beta}(U)$ for every $\beta<\alpha$.

The proof is obtained in two steps: first we compute the derivatives of $u$ by means of Lemma 4.2 and then we study the regularity of this term, which is explicitly known.

Step 1. In order to differentiate $v$, we use the representation formula proved in Theorem 4.3, The function

$$
\xi \rightarrow I_{1}(\xi, \bar{\xi})=\int \Gamma_{\bar{\xi}}(\xi, \zeta) N_{1}(\zeta, \bar{\xi}) d \zeta+\sum_{|\rho|,|\theta| \leq 1} \int e_{\rho} D_{\bar{\xi}, \theta} \Gamma_{\bar{\xi}}(\xi, \zeta) N_{2, m, \theta, \rho}(\zeta, \bar{\xi}) d \zeta
$$

is of class $C_{L}^{m, \beta}$. Let us study

$$
I_{2}(\xi, \bar{\xi})=\sum_{|\rho|,|\theta| \leq 1} \int e_{\rho} D_{\bar{\xi}, \theta} \Gamma_{\bar{\xi}}(\xi, \zeta) N_{2, m, \theta, \rho}(\zeta, \bar{\xi}) d \zeta
$$

We fix a function $\psi$ in $C^{\infty}(R)$ satisfying $0 \leq \psi \leq 1, \psi(\tau)=0 \forall \tau \leq 1$ and $\psi(\tau)=1 \forall \tau \geq 1$, and define, for every $\epsilon>0$,

$$
I_{2, \varepsilon}(\xi, \bar{\xi})=\sum_{|\rho|,|\theta| \leq 1} \int \psi\left(\frac{d_{\bar{\xi}}(\xi, \zeta)}{\epsilon}\right) e_{\rho} D_{\bar{\xi}, \theta} \Gamma_{\bar{\xi}}(\xi, \zeta) N_{2, m, \theta, \rho}(\zeta, \bar{\xi}) d \zeta
$$

Then it is not difficult to show that

$$
\sup _{\delta_{\bar{\xi}}(\xi, \bar{\xi})<\varepsilon}\left|I_{2, \epsilon}(\xi)-I_{2}(\xi, \bar{\xi})\right| \leq C \epsilon^{m+\beta} .
$$

Finally, if for $|\sigma|=m$ we set

$$
I_{2}^{\sigma}(\bar{\xi})=\sum_{|\rho|,|\theta| \leq 1} \int D_{\sigma}\left(e_{\rho} D_{\bar{\xi}, \theta} \Gamma_{\bar{\xi}}\right)(\xi, \zeta) N_{2, m, \theta, \rho}(\zeta, \bar{\xi}) d \zeta
$$

then we obtain

$$
\sup _{\delta_{\bar{\xi}}(\xi, \bar{\xi})<\varepsilon}\left|D_{\sigma} I_{2, \epsilon}(\xi, \bar{\xi})-I_{2}^{\sigma}(\bar{\xi})\right| \leq C \epsilon^{\beta} .
$$

All the hypotheses of Lemma 4.2 are satisfied. Hence the derivative $D_{\sigma} u$ exists for every $\sigma$ of weight $m$.

Step 2. The proof of the Hölder continuity of derivatives is organized in two parts. First, using the Hölder estimates provided in Theorem 4.3 and arguing as in [C], we obtain that the derivatives $D_{\sigma} u$, with $|\sigma|=m$, are of class $C_{L}^{\beta}$ for $\beta<\alpha$. Then, by using the estimates of Theorem 4.4 and arguing as in [M], we obtain that for every $\sigma$ such that $|\sigma|=m-1$, the derivatives $D_{\sigma} u$ belong to $C_{(3)}^{(1+\beta) / 2}(U)$ for every $\beta<\alpha$. These two estimates together imply that $u \in C_{L}^{m, \beta}(U)$ for every $\beta<\alpha$.

In view of the iteration we recall that if $u$ is a solution of (1.12), then its derivatives satisfy the same equation, with a different second member (see [M] for a detailed proof of the following lemma). 
Lemma 4.6. If $u$ is a solution of (1.12), then the function $v_{8}=\partial_{t} u$ is a solution of $L v_{8}=\partial_{t} g+F_{8, c}$, where

$$
F_{8, c}=-2 \partial_{t} a X \partial_{t} u-2 \partial_{t} b Y \partial_{t} u+\partial_{t} h \partial_{x_{3}} u+\partial_{t} c \partial_{t} u .
$$

The function $\widetilde{v}_{3}=\partial_{x_{3}} u$ is a solution of $L \widetilde{v}_{3}=\partial_{x_{3}} g+F_{3, c}$, where

$$
F_{3, c}=-2 \partial_{x_{3}} a X \partial_{t} u-2 \partial_{x_{3}} b Y \partial_{t} u+\partial_{x_{3}} h \partial_{x_{3}} u+\partial_{x_{3}} c \partial_{t} u .
$$

The function $v_{1}=X u$ is a solution of $L v_{1}=X g+F_{1, c}$, where

$$
\begin{gathered}
F_{1, c}=Y\left(q \partial_{t} u\right)+q \partial_{t} Y u+\frac{h}{1+u_{t}^{2}}\left(Y \partial_{x_{3}} u-X \partial_{x_{3}} u \partial_{t} u\right) \partial_{t} u \\
-\frac{q \partial_{t} u-c}{1+u_{t}^{2}}\left(Y \partial_{t} u-X \partial_{t} u \partial_{t} u\right) \partial_{t} u-X h \partial_{x_{3}} u+X\left(q \partial_{t} u\right) \partial_{t} u-c \partial_{t} u,
\end{gathered}
$$

and $q=h \partial_{x_{3}} u-(X a+Y b) \partial_{t} u-g$.

Finally, the function $v_{2}=Y u$ is a solution of $L v_{2}=Y g+F_{2, c}$, where

$$
\begin{gathered}
F_{2, c}=X\left(q \partial_{t} u\right)+q \partial_{t} X u-\frac{h}{1+u_{t}^{2}}\left(X \partial_{x_{3}} u+Y \partial_{x_{3}} u \partial_{t} u\right) \partial_{t} u \\
+\frac{q \partial_{t} u-c}{1+u_{t}^{2}}\left(X \partial_{t} u+Y \partial_{t} u \partial_{t} u\right) \partial_{t} u-Y h \partial_{x_{3}} u+Y\left(q \partial_{t} u\right) \partial_{t} u-c \partial_{t} u .
\end{gathered}
$$

\section{The NONLINEAR OPERATOR}

In this section we prove Theorem 1.1. We will recognize that the derivatives of the function $u$ satisfy a linear equation, and we will apply to it the results of the preceding section.

Let us fix a solution $u$ of class $C_{\text {euclid }}^{3, \alpha}(\Omega)$ of equation (1.4), let us define $a, b$ in terms of the solution $u$ as in (1.2), and let us choose

$$
c=k u_{t} \frac{\left(1+a^{2}+b^{2}\right)^{3 / 2}}{\left.1+u_{t}^{2}\right)^{1 / 2}},
$$

and let $h=h(a, b, \cdot)$ be the function defined in the introduction. Hence $a, b, c$ and $h$ are of class $C_{\text {euclid }}^{2, \alpha}(\Omega)$. Let $X=X_{u}, Y=Y_{u}, S=S_{u}$ be the vector fields defined in (1.11) in terms of $a, b, c$ and $h$, and let

$$
L=L_{u}=X^{2}+Y^{2}+S \text {. }
$$

We recall (see for example $[\mathbf{C}]$ ) that $a=Y u$ and $b=-X u$, so that

$$
\begin{gathered}
X a+Y b=X Y u-Y X u=-\frac{\mathcal{L} u}{1+u_{t}^{2}} \partial_{t} u \\
=\frac{k\left(1+a^{2}+b^{2}\right)^{3 / 2}\left(1+u_{t}^{2}\right)^{1 / 2}+h(a, b, \cdot) \partial_{x_{3}} u}{1+u_{t}^{2}} \in C_{\text {euclid }}^{2, \alpha}(\Omega), \\
X b-Y a=-\left(X^{2} u+Y^{2} u\right)=-\frac{\mathcal{L} u}{1+u_{t}^{2}} \in C_{\text {euclid }}^{2, \alpha}(\Omega) .
\end{gathered}
$$

Hence the operator $L$ satisfies all the assumptions of Theorem 1.2 . 
We will denote by $C_{L_{u}}^{m, \alpha}$ the classes of Hölder continuous functions introduced in Definition 2.4 We will also set $\widetilde{D}_{1}=X, \widetilde{D}_{2}=Y, \widetilde{D}_{3}=\partial_{x_{3}}$ and $\widetilde{D}_{8}=\partial_{t}$. For every multi-index $\sigma=\left(\sigma_{1}, \ldots, \sigma_{m}\right)$, with $\sigma_{r} \in\{1,2,3,4\}$, let

$$
\widetilde{D}_{\sigma}=\widetilde{D}_{\sigma_{1}} \cdots \widetilde{D}_{\sigma_{m}}
$$

We will call the number $m$ the length of $\sigma$, and we will write $l(\sigma)=m$. Moreover, we will denote by $\widetilde{D}^{m}$ the vector whose components are all the derivatives of length at most $m$.

By (1.3) and (1.5), $u$ is a solution of

$$
X^{2} u+Y^{2} u-(X a+Y b) \partial_{t} u-h(a, b, \cdot) \partial_{x_{3}} u=k\left(1+a^{2}+b^{2}\right)^{3 / 2}\left(1+u_{t}^{2}\right)^{1 / 2},
$$

and in the following proposition we will show that its derivatives are solution of an equation associated to the operator $L$.

Proposition 5.1. Let $m$ be a natural number and let $\sigma$ be a multi-index with $\sigma_{i} \in$ $\{1,2,3,8\}$ and $l(\sigma)=m$. Assume that $D^{m} u \in C_{L_{u}}^{2, \beta}(\Omega)$ with $\left.\beta \in\right] 0,1[$. Then the function

$$
v_{\sigma}=\widetilde{D}_{\sigma} u
$$

is a solution of

$$
L\left(v_{\sigma}\right)=f_{\sigma}, \text { where } f_{\sigma}=f_{\sigma}\left(\widetilde{D}^{m} u, X \widetilde{D}^{m} u, Y \widetilde{D}^{m} u\right),
$$

and $f_{\sigma}$ is a smooth function of its arguments.

Proof. Let us prove the assertion by induction on $m$. By Lemma4.6 it follows that the functions $v_{i}$ with $i \in\{1,2,3,8\}$ are solutions of

$$
\begin{gathered}
\left(X^{2}+Y^{2}-(X a+Y b) \partial_{t}+h \partial_{x_{3}}\right) v_{i}=\widetilde{D}_{i}\left(k\left(1+a^{2}+b^{2}\right)^{3 / 2}\left(1+u_{t}^{2}\right)^{1 / 2}\right)+F_{i, 0} \\
=\widetilde{D}_{i}\left(k\left(1+a^{2}+b^{2}\right)^{3 / 2}\right)\left(1+u_{t}^{2}\right)^{1 / 2} \\
+k u_{t} \frac{\left(1+a^{2}+b^{2}\right)^{3 / 2}}{\left(1+u_{t}^{2}\right)^{1 / 2}}\left[\widetilde{D}_{i}, \partial_{t}\right] u+k u_{t} \frac{\left(1+a^{2}+b^{2}\right)^{3 / 2}}{\left(1+u_{t}^{2}\right)^{1 / 2}} \partial_{t}\left(\widetilde{D}_{i} u\right)+F_{i, 0},
\end{gathered}
$$

where the $F_{i, 0}$ are defined in Lemma 4.6. Bringing to the left hand side the second term and using the definition of $c$, we get

$$
L\left(v_{i}\right)=\widetilde{D}_{i}\left(k\left(1+a^{2}+b^{2}\right)^{3 / 2}\right)\left(1+u_{t}^{2}\right)^{1 / 2}+k u_{t} \frac{\left(1+a^{2}+b^{2}\right)^{3 / 2}}{\left(1+u_{t}^{2}\right)^{1 / 2}}\left[\widetilde{D}_{i}, \partial_{t}\right] u+F_{i, 0} .
$$

The right hand side depends on

$$
\widetilde{D} u, X \widetilde{D} u, X \widetilde{D} u, \partial_{t} a, \partial_{t} b, \partial_{x_{3}} a, \partial_{x_{3}} b,
$$

and for every $i \in\{3,8\}$, it has been proved in $[\mathrm{M}]$ that

$$
\widetilde{D}_{i} a=\widetilde{D}_{i} Y u=\frac{Y \widetilde{D}_{i} u-\partial_{t} u X \widetilde{D}_{i} u}{1+u_{t}^{2}}, \quad \widetilde{D}_{i} b=-\widetilde{D}_{i} X u=-\frac{X \widetilde{D}_{i} u+\partial_{t} u Y \widetilde{D}_{i} u}{1+u_{t}^{2}} .
$$

It is then clear that $v_{i}=\widetilde{D}_{i} u$ is a solution of the required equation, and the thesis is proved for $m=1$. 
Let us assume that the thesis is true for $m$ and prove it for $m+1$. Let $l(\sigma)=m$, and let $v_{\sigma}$ be a solution of $L\left(v_{\sigma}\right)=f_{\sigma}$. By Lemma 4.6. for every $i \in\{1,2,3,8\}$ the function $\widetilde{D}_{i} v_{\sigma}$ is a solution of

$$
L\left(v_{\sigma}\right)=\widetilde{D}_{i}\left(f_{\sigma}\right)+F_{i, c},
$$

and the thesis follows as before, by commuting the derivative $\widetilde{D}_{i}$ with the vector fields $X$ and $Y$.

Proof of Theorem 1.1. We will prove that

$$
\widetilde{D}^{m} u \in C_{L_{u}}^{7, \beta}(\Omega),
$$

for every $m \in \mathbb{N}$ and for every $\beta<\alpha$, which obviously proves the statement.

Since $u \in C_{\text {euclid }}^{3, \alpha}(\Omega)$, then for every $i \in\{1,2,3,8\}$ we have $\widetilde{D}_{i} u \in C_{\text {euclid }}^{2, \alpha}(\Omega)$, and by Proposition 5.1]

$$
L\left(\widetilde{D}_{i} u\right)=f_{i}\left(\widetilde{D}^{1} u, X \widetilde{D}^{1} u, Y \widetilde{D}^{1} u\right) \in C_{L_{u}}^{1, \alpha}(\Omega) .
$$

Hence we can apply Theorem 4.5 with $m=3$, and we get $\widetilde{D}_{i} u \in C_{L_{u}}^{3, \beta}(\Omega)$. In particular, $u \in C_{L_{u}}^{4, \beta}(\Omega), a, b, h, c \in C_{L_{u}}^{3, \beta}(\Omega)$, and $f_{i}\left(\widetilde{D}^{1} u, X \widetilde{D}^{1} u, Y \widetilde{D}^{1} u\right) \in C_{L_{u}}^{2, \beta}(\Omega)$. Hence we can apply Theorem 4.5 with $m=4$ to the function $\widetilde{D}_{i} u \in C_{L_{u}}^{3, \beta}(\Omega)$, to get $\widetilde{D}_{i} u \in C_{L_{u}}^{4, \beta}(\Omega)$, for every $i \in\{1,2,3,8\}$. Iterating this procedure, we get

$$
\widetilde{D}_{i} u \in C_{L_{u}}^{7, \beta}(\Omega),
$$

for every $i$ and for every $\beta<\alpha$. This prove the thesis for $m=1$. Now we assume that the thesis is true for $m$, and we prove it for $m+1$. By the inductive hypothesis the function $\widetilde{D}^{m} u$ is of class $C_{L_{u}}^{7, \beta}(\Omega)$. Hence, for any fixed $\sigma$ of length $m+1$ the function $v_{\sigma}=\widetilde{D}_{\sigma} u$ is of class $C_{L_{u}}^{4, \beta}(\Omega)$ and, by Proposition 5.1 is a solution of the equation

$$
L v_{\sigma}=f_{\sigma}\left(\widetilde{D}^{m} u, X \widetilde{D}^{m} u, Y \widetilde{D}^{m} u\right) .
$$

Since the second member is of class $C_{L_{u}}^{3, \beta}(\Omega)$, by Theorem 4.5 $v_{\sigma}$ is $C_{L_{u}}^{5, \beta}(\Omega)$, for every $\sigma$ of length $m+1$. In other words, $\widetilde{D}^{m} u$ is of class $C_{L_{u}}^{5, \beta}(\Omega)$, and the second member $f_{\sigma}$ of (5.2) is of class $C_{L_{u}}^{4, \beta}(\Omega)$. Hence, by Theorem $4.5 \widetilde{D}^{m} u$ is of class $C_{L_{u}}^{6, \beta}(\Omega)$, and $f_{\sigma}$ is of class $C_{L_{u}}^{5, \beta}(\Omega)$. Finally, always by Theorem $4.5 v_{\sigma} \in C_{L_{u}}^{7, \beta}(\Omega)$.

\section{REFERENCES}

[BG] E. Bedford, B. Gaveau, Envelopes of holomorphy of certain 2-spheres in $C^{2}$, Amer. J. Math., 105, 1983, 975-1009. MR 84k:32016

[BK] E. Bedford, W. Klingenberg, On the envelopes of holomorphy of a-spheres in $C^{2}$, Journal of the A.M.S. , 4, 3, 1991, 623-646. MR 92j:32034

[CS] E. M. Chirka, Shcherbina, Pseudoconvexity of rigid domains and foliation of hulls of graphs, Ann. Scuola Norm. Sup. Pisa Cl. Sci. (4), 21, 1995, 707-735. MR 98f:32016

[C] G. Citti, $C^{\infty}$ regularity of solutions of a quasilinear equation related to the Levi operator, Ann. Scuola Norm. Sup. Pisa, Cl. Sci. (4), 23, 3, (1996), 483-529. MR 98b:35072

[Csem] G. Citti, Regolarità di grafici non Levi-piatti, Seminario di Analisi, Dip. Mat. Univ. Bologna (A.A. 1996/97), Tecnoprint, Bologna, 186-195.

[CM1] G. Citti, A. Montanari, Analytic estimates of solutions of the Levi equation, J. Differential Equations, 173, (2001), 356-389.

[CM2] G. Citti, A. Montanari, $C^{\infty}$ regularity of solutions of an equation of Levi's type in $R^{2 n+1}$, Annali di Matematica Pura Appl. (4), 180, (2001), 27-58 CMP 2001:11 
[CPP] G. Citti, A. Pascucci, S. Polidoro, On the regularity of solutions to a nonlinear ultraparabolic equation in mathematical finance, Diff. Int. Eq, 14 (2001), 701-738.

[F] G. B. Folland, Subelliptic estimates and function spaces on nilpotent Lie groups, Arkiv Mat., 13, 1975, 161-207. MR 58:13215

[FS] G. B. Folland, E. M. Stein, Estimates for the $\bar{\partial}_{b}$ Complex and Analysis on the Heisenberg Group, Comm. Pure Appl. Math. 20, 1974, 429 - 522. MR 51:3719]

[FL] B. Franchi, E. Lanconelli, Hölder regularity theorem for a class of linear nonuniformly elliptic operators with measurable coefficients, Ann. Scuola Norm. Sup. Pisa Cl. Sci. (4), 10, 1983, 523-541. MR 85k:35094

[H] L. Hörmander Hypoelliptic second order differential equations Acta Math., 119, 1967, 147-171. MR 36:5526

[HK] G. Huisken, W. Klingenberg, Flow of real hypersurfaces by the trace of the Levi form, Mathematical Research Letters 6 (1999), 645-662. MR 2001f:53141

[K] W. Klingenberg, Real hypersurfaces in Kähler manifolds preprint.

[M] A. Montanari, Real hypersurfaces evolving by Levi curvature: smooth regularity of solutions to the parabolic Levi equation, to appear in Comm. Partial Differential Equations 26 (9\&10) (2001) 1633-1664.

[NSW] A. Nagel, E. M. Stein, S. Wainger Balls and metrics defined by vector fields I: Basic properties. Acta Math. 155, 1985, 103-147. MR 86k:46049

[RS] L. Rothschild, E. M. Stein Hypoelliptic differential operators and nilpotent groups Acta Math. 137, 1977, 247-320. MR 55:9171

[Sc] N. V. Shchcerbina, On the polynomial hull of a graph. Indiana Univ. Math J., 42, 1993, 477-503. MR 95e:32017

[ST1] Z. Slodkowski, G. Tomassini, Weak solutions for the Levi equation and Envelope of Holomorphy, J. Funct. Anal, 101, no. 4, 1991, 392-407. MR 93c:32018

[ST2] Z. Slodkowski, G. Tomassini, Evolution of subsets in $\mathbb{C}^{2}$ and a parabolic problem for the Levi equation, Ann. Scuola Norm. Sup. Pisa, Cl. Sci. (4), 25, (1997), 757-784. MR 2000c:32013

[ST3] Z. Slodkowski, G. Tomassini, Evolution of special subsets of $\mathbb{C}^{2}$, Adv. Math. 152, (2000), no. 2, 336-358. MR 2001i:32019

[ST4] Z. Slodkowski, G. Tomassini, Evolution of a graph by Levi form, Gulliver, Robert (ed.) et al., Differential geometric methods in the control of partial differential equations. (Boulder, CO, 1999), Providence, RI: American Mathematical Society (AMS). Contemp. Math. 268, 373-382 (2000). CMP 2001:07

Dipartimento di Matematica, Universita di Bologna, Piazza di Porta S. Donato 5, 40127, Bologna, Italy

E-mail address: citti@dm.unibo.it

Dipartimento di Matematica, Universita di Bologna, Piazza di Porta S. Donato 5, 40127, Bologna, ITALY

E-mail address: montanar@dm.unibo.it 